\title{
Estimation of drug-likeness properties of GC-MS separated bioactive compounds in rare medicinal Pleione maculata using molecular docking technique and SwissADME in silico tools
}

\author{
Hakani D. Sympli ${ }^{1}$ (1)
}

Received: 6 August 2020 / Revised: 26 November 2020 / Accepted: 28 November 2020 / Published online: 24 February 2021

(c) The Author(s), under exclusive licence to Springer-Verlag GmbH, AT part of Springer Nature 2021

\begin{abstract}
The main aim of the paper was to determine bioactive compounds in Pleione maculata extracts using gas chromatographic technique and to investigate their drug-likeness potential using molecular docking algorithm and ADME studies on the recent intractable disease, for example, SARS-CoV-2. Pleione maculata sample was prepared for GC-MS analysis. The peak components were identified based on the NIST Library. Molecular docking was performed using PatchDock, and energy refinement was carried out using the FireDock algorithm followed by drug-likeness analysis using the SwissADME tool. The mass spectrum revealed various pharmacologically important compounds and novel compounds 8-oxatetracyclo $\{5.2 .1 .1(2,6)$. 1(4,10)\}dodecane, 7-tert-butyl-1,9,9-trimeth, docosane, 2,4-dimethyl, kryptogenin 2,4-dinitrophenyl hydrazine, and $N$-decylalpha, $D$-2-deoxyglycoside which are reported for the first time. Molecular docking using PatchDock illustrates GC-MS compounds Nor-diazepam, $3-\{N$-hydroxymethyl $\}$ aminocarbonyloxy a good docking and high binding affinity with atomic contact energy $-10.95 \mathrm{kcal} / \mathrm{mol}$ against SARS-CoV-2 spike protein S2 subunit. ADME analysis predicts Nor-diazepam,3$\{\mathrm{N}$-hydroxymethyl $\}$ aminocarbonyloxy and andrographolide showed very high drug-likeness parameters with no metabolism disturbances. The random control antiviral drug arabidiol revealed a lower binding affinity and lower solubility compared to bioactive compounds of $P$. maculata. The study depicts the first and novel report on various pharmaceutical important GC-MS bioactive compounds and molecular docking study on Pleione maculata having potential against various intractable diseases.
\end{abstract}

Keywords Gas chromatography $\cdot$ SARS-CoV-2 $\cdot$ Pleione maculata $\cdot$ Molecular docking $\cdot$ Drug-likeness

\section{Introduction}

A worldwide viral outbreak of dreadful disease COVID-19 arose during December 2019 in Wuhan, China (Yang and Wang 2020; Yang et al. 2020). Severe acute respiratory syndrome coronavirus-2 (SARS-CoV-2) as named by the International Committee on Taxonomy of Viruses (ICTV) and diseases cause was coronavirus disease 2019 (COVID-19). COVID-19 is no new viruses but a possible mutation of the long-known SARS-CoV-1. A genome variation analysis was analyzed using a detective genome computational tool, and it

Hakani D. Sympli

symplihakani@gmail.com

1 Department of Biotechnology, School of Biosciences, Assam Don Bosco University, Tapesia, Kamarkuchi, Sonapur, Assam 782402, India revealed SARS-CoV-2 shares $80 \%$ similarities with the gene pool of SARS-CoV-1 (Zhang et al. 2017) with nearly 17\% variation which was a mutation occurring in spike protein and envelope protein (Sardar et al. 2020). The viral genome analysis of the SARS-CoV-1 open reading frame (orf) was observed to have a potential mutation to adapt to a new environment (Groneberg et al. 2005) and recombination ( $\mathrm{Li}$ Fang 2016) which might cause severe virulence of the virus. The severe acute respiratory syndrome (SARS) is a crownlike virus which was spread widely in late 2000 over 25 countries causing thousands of cases and death (Wen et al. 2011). Since 2003, antiviral research has been evaluated for an anti-SARS-COV-1 activity to prevent re-emergence of the disease. The genome of SARS-CoV-1 encodes various vital target proteins: spike protein (S), 3CL protease, NTPase/helicase, RNA-dependent RNA polymerase, membrane protein (M), an envelope protein (E), and nucleocapsid phosphoprotein $(\mathrm{N})$ which takes part in virus replication, transcription, 
and translation (Yang and Wang 2020). The main molecule that mediates coronavirus entry into a host cell is their spike protein (S) which is multi-functional (Fang 2016), where the attachment is initiated by the $\mathrm{S} 1$ subunit and conformation changes took place from pre-fusion to post-fusion or membrane fusion form. The virus incorporation is initiated by the S2 subunit of spike glycoprotein through membrane fusion with the host receptor (Rane et al. 2020). The viral 3-chymotrypsin-like protease responsible for replication complex (Anand et al. 2003) is considered highly conserved between SARS-CoV-1 and SARS-CoV-2 (Zhang et al. 2017; Donald and Hai-Feng 2020). The SARS-CoV-1 spike protein has a strong binding affinity towards human receptor angiotensinconverting enzyme 2 (ACE2) based on structure and interaction (Zhang et al. 2020a, b). The main agent for transmission is through respiratory droplets and can be transmitted from human to human through contact with droplets (Yang and Wang 2020). For early diagnosis of SARS-CoV-1, improved RT-PCR assays were carried out which is used worldwide for virus identification and its high specificity (Shen et al. 2020). The symptoms of SARS-CoV-1 were persistent fever, chills, dry cough, dizziness, headache, sore throat, sputum production, vomiting, and nausea; special attention was given for watery diarrhea, but the primary target for infection was respiratory epithelial cells. The viral effect immune-mediated mechanism and molecular studies showed epithelial cells of the gastrointestinal tract to be major target cells (Groneberg et al. 2020). In 2019, various health authorities of Hubei Province, China, reported novel COVID19 disease as pneumonia (Wu et al. 2020) or COVID-19 pneumonia (Tian et al. 2020). Pneumonia is a type of fatal respiratory tract infection that is caused by either bacteria (Streptococcus pneumonia) or viruses, and symptoms are no different from the deadly virus SARS-CoV-2 such as high fever, shortness of breath, rapid breathing, and cough (Zafar 2016). Earlier, SARS-CoV-1 patients were treated with antiinflammatory steroidal compounds such as methylprednisolone (Groneberg et al. 2005; Wu et al. 2017), and metabolite profiling for SARS-CoV-1 survivors was carried out after 12 years of recovery using ultra-high-performance liquid chromatography-mass spectrometry (UPLC-MS) and gas chromatography-mass spectrometry (GC-MS); a significant portion $(64 \%)$ of recovered patients were prone to lung infections and various serum metabolic disorders associated with lipid metabolism including hyperlipidemia (HL), cardiovascular abnormality (CVA), and an abnormality in glucose metabolism (AGM). Single-stranded antisense as an antiviral compound has been a vital therapeutic area for emerging viruses (Gulam et al. 2016). Antisense therapy (antisense antivirals) treats diseases using single-stranded antisense oligonucleotides to target specific mRNA sequences and block translation of viral protein (Gulam et al. 2016) or modifies protein expression (Sharad 2019). To date, there are a lot of controversies regarding vaccine and drug development against SARS-CoV-2 pneumonia, though various antiviral drugs are being used for treatment there appeared to be many disadvantages and side effects caused. The transmission from person to person is highly contagious due to inadequate global healthcare facilities (Panda et al. 2020). Plants are the main source of natural medicines as they produce various biologically active secondary metabolites. Secondary metabolites provide nutritional and beneficial effects on human health (Lakshmi and Rajalakshmi 2011). According to World Health Organization (WHO), about 80-90\% population relies on traditionally prepared medicinal plants for regular health care as it is safe and ready to use (Rizvi and Misra 2013; Ekor 2014). Orchids are one large kingdom of plants that are overexploited and also climatic changes; their illegal trading led to extinction and biodiversity loss (Pant 2003). About 50\% of orchids are being used in traditional medicines apart from being sources of ornaments (Tsering et al. 2017). Epiphytic orchid growing on other living or non-living materials for physical supports tends to release bioactive secondary metabolites when they are exposed to disturbance (Lindley and Paxton 1851). Pleione maculata commonly known as peacock orchid is a rare unexplored epiphyte on the verge of extinction (Chauhan and Sharma 2017) growing on trees at a high elevations of about 600-1600 m (Lindley and Pacton 1851). The epiphyte is a well-known medicinal plant in the northeastern region of India, where local people use pseudobulbs or rhizomes to treat liver problems, stomach ailments, and headaches (Pant 2003; Teoh 2016).

Today, in silico studies are more favored for drug identification to find the interaction of the pharmaceutically important compounds with their targets. The most commonly known computational tool for drug target is molecular docking using various algorithms. PatchDock is highly efficient (Prabhu and Rajeswari 2016) accurate (Doss et al. 2014), fast transformational search, and free-online server in comparison to other computational molecular docking servers. PatchDock is a molecular docking algorithm based on the principle of shape complementarity. A complementarity molecular shape is a yield forming a conformational transformation of each docking complex known as induced fit. The transformed molecules can be further evaluated based on the scoring function which involves geometric-fit and binding affinity based on atomic contact energy (ACE). PatchDock calculates the amount of atomic contact energy (ACE) which is an atomic desolvation free energy required to replace ligand molecule from water contact to protein contact (Guo et al. 2012; Maiti and Banerjee 2020). PatchDock algorithms contain three stages, (a) molecular shape representation based on geometric patches (concave, convex, and flat surface pieces), (b) surface patch matching, and (c) filtering and scoring (Duhovny et al. 2002). There are various parameters involved for running a docking 
interaction between molecules such as root mean square deviation (RMSD) and a complex type. The RMSD is applied to prevent redundancy of molecule and is exact (Duhovny et al. 2002). PatchDock uses techniques such as geometry hashing and pose clustering which detects advance data structures and spatial pattern. In docking, energy refinement is required for further development of drug compounds for example FireDock (Andrusier et al. 2007; Surana et al. 2018). FireDock (fast iteration refinement in molecular docking) refinement of energy uses Monte Carlo binding score minimization, is highly efficient and easy to understand, and requires no prior knowledge in docking (Lipinski et al. 1997). The scores are atomic contact energy (ACE), softened van der Waals interaction, partial electrostatics, and additional estimation of binding energy. FireDock algorithm includes three refinement steps: (1) side-chain optimization, (2) rigid-body minimization, and (3) scoring and ranking (Lipinski et al. 1997). The docking analysis can be predicted base on higher binding affinity and lowest docked energy (Iyamah et al. 2017). Before clinical studies, the drug-likeness of compounds can be analyzed using computational tools that are cost-effective and less tedious. SwissADME is a free web tool available to evaluate pharmacokinetics based on different drug-likeness parameters such as physicochemical properties, solubility, and pharmacokinetics of molecule (Daina et al. 2003).

A compound separation technique gas chromatography-mass spectrophotometry will provide ideas on different bioactive compounds present in plants. Gas chromatography is an instrumental technique coupled with mass spectrometry applied for separation, identification, and quantification of organic compounds and chemical mixtures study. An inert gas such as helium is used as a mobile phase (carrier gas). The samples to be analyzed will be injected and interacted with glass or metal columns coated with the stationary phase and elute different compounds at the different times called retention time (Ghosal and Srivastava 2013). The GC-MS method is highly sensitive, reproducible, and high-speed resolution (Dua and Garg 2013). The study aimed to exploit safe, medicinal, nutraceutical effective natural compounds from Pleione maculata using GC-MS analysis as there seems to be an unreached target globally requiring active research against SARS-COV-2. The selected GC-MS compounds for docking were based on their bioactivity against symptoms related to SARS-CoV-2 pneumonia such as antiinflammatory activity, preventing cardiac insufficiency, preventing fatigue, preventing shortness of breath, preventing gastrointestinal diseases, heartbeat improvement, antiviral activities, and a compound having repellent activity, larvicidal activity, based on earlier knowledge in using an antimalarial drug for reducing a viral load (Rane et al. 2020). In earlier years, steroidal compound and antisense target were also used for the treatment of viral diseases
(Chidambaram et al. 1996; Kim et al 2009; Chen et al. 2014); therefore, with this prior knowledge, GC-MS analysis of Pleione maculata identified steroidal antiviral and an antisense target compound as a key compound to perform docking. The paper also evaluates the drug-likeness potential of compounds possessing multi-target bioactivity using a molecular docking algorithm to observe high binding affinity and conformational fit between bioactive compounds of $P$. maculata against target proteins of SARS-CoV-2. A random control antiviral drug arabidiol was used in severe cases of COVID-19 as it was observed to reduce the viral disease (Wang et al. 2020).

\section{Materials and methods}

\subsection{Collection and extract preparation of plant material}

Pleione maculata sample was collected from dense forest of Khliehriat, East Jaintia Hills regions of Meghalaya, and was processed. The parts of P. maculata were washed under running tap water, surface-sterilized with distilled water, $1 \%$ sodium hypochlorite, and re-washed with distilled water. The samples were shade-dried and crushed, and each plant part was soaked in three different solvents (ethanol, methanol, acetone) followed by $24-48$-h incubation. The crude extract was filtered using Whatman filter paper Grade No-1 with circle size of $125 \mathrm{~mm}$ diameter (Cat No 1001125) (Vijisaral and Arumugam 2014). The solvent extracts are then evaporated using open-air evaporation in a laminar airflow hood for $24 \mathrm{~h}$ and centrifuged at $12,000 \mathrm{rpm}$ for about $15 \mathrm{~min}$ at $4{ }^{\circ} \mathrm{C}$. The concentrated extracts were transferred into a micro-centrifuge tube for GC-MS analysis.

\subsection{GC-MS analysis}

Gas chromatography-mass spectrometry of samples was analyzed in IIT Guwahati Biotech Park, Assam, using PerkinElmer (USA). The GC-MS system model was Clarus 680 GC \& Clarus $600 \mathrm{C}$ MS comprising a liquid autosampler with aid of Turbo mass ver. 5.4.2 software. The chromatography was performed on a capillary column Elite of $5 \mathrm{MS} 60.0 \mathrm{~m} \times 250 \mu \mathrm{m}$, ID of $0.25 \mathrm{~mm}$, and film thickness of $0.25 \mu \mathrm{m}$, and stationary phase was $5 \%$ diphenyl $95 \%$ dimethylpolysiloxane. The injection volume of the sample was $1 \mu \mathrm{l}$ with a 0:1 split ratio. Helium (99.999\%) was used as carrier gas throughout the column. The solvent delay was by $9.00 \mathrm{~min}$, and transfer temperature was $200{ }^{\circ} \mathrm{C}$. The injector and ion source temperature were $280{ }^{\circ} \mathrm{C}$ and 
$180{ }^{\circ} \mathrm{C}$, respectively. The initial temperature in the oven was programmed from $110{ }^{\circ} \mathrm{C}$ for $3 \mathrm{~min}$ (Thomas et al. 2013), ramp $5{ }^{\circ} \mathrm{C} / \mathrm{min}$ to $200{ }^{\circ} \mathrm{C}$ and hold for $3 \mathrm{~min}$ and was again increased for $5{ }^{\circ} \mathrm{C} / \mathrm{min}$ to $300^{\circ} \mathrm{C}$ and hold for $10 \mathrm{~min}$ (Darmasiwi et al. 2015). The sample was scanned from 40 to $600 \mathrm{Da}$.

\subsection{Identification of components using NIST}

The peaks were analyzed using the data analysis software NIST-2008. The National Institute of Standards and Technology (NIST) is a mass spectral search database for comparisons of the acquired and unknown spectrum with NIST/EPA (Environmental protection Agency)/NIH (National Institute of Health) databases. The components were identified based on standards employed by the National Institute Standards and Technology (NIST) Library (Darmasiwi et al. 2015). The detection was employed by comparing peaks with that of mass spectral standard reference data NIST having more than 62,000 patterns. A detailed chemical structure of the GC-MS graph of a molecule was presented using ChemDraw Ultra 8.0.3 by CambridgeSoft (www.cambridgesoft.com) (CS ChemOffice-Drawing, Modelling, and Information).

\subsection{Molecular docking using PatchDock}

\subsubsection{Input}

Protein structure retrieval: Protein receptor target was retrieved from RCSB (Research Collaboratory for Structural Bioinformatics) Protein Data Bank (PDB) (https://www. rcsb.org/) in 3D structure as shown in Fig. 1. The following three target receptors were (1) SARS-CoV-2-3CL protease
(PDB ID: 6M2Q), (2) SARS-CoV-2 RNA-dependent RNApolymerase (PDB ID: 6M71), and (3) SARS-CoV-2 spike glycoprotein receptor $\mathrm{S} 2$ subunit (PDB ID: 6LXT).

\subsubsection{Ligand retrieval}

A total of 19 ligand molecules as shown in Fig. 2 were retrieved from PubChem (http://pubchem.ncbi.nlm.nih. gov/) database in SDF file format. The small ligand in SDF format was converted into PDB format using PyMOL a molecular modeling package (The PyMOL Molecular Graphics System, Version System, version 1.7.4 Schrödinger, LLC) (Prabhu and Rajeswari 2016; Yadav et al. 2017). The compound ID of the 3D structures of ligands was CID: 6211 (2,4,6-pyrimidinetrione), CID: 248,856 (21-acetoxypregenelone), CID: 543,946 (P-menth-8(10)-en-9-ol), CID: 541,761 (Nor-diazepam, 3-N-hydroxymethyl,aminocarbonyloxy), CID: 441,207 (digitoxin), CID: 19,089,489 (DI- $N$-decylsulfone), CID: 3893 (14-dodecanoic acid), CID: 13,948 (estra,13,5 (10)-trien-17-beta-ol), CID: 5,283,405 (arachidonic amide, $N$-5-hydroxy- $N$-pentyl), CID: 572,031 (1-methylsulfanyl-9,10-dioxo-9,10-dihydro-anthracene-2-carboxylic acid), CID: 14,077,841 (cholesterol margarate), CID: 8215 (docosanoic acid), CID: 99,470 (26-hydroxy cholesterol), CID: 5,318,517 (andrographolide), CID: 135,426,867 (6H-purin-6-one-,1,7-dihydro-2-methylamino), CID: 261,799 (pseduosarsasapogenin), CID: 71,360,559 (oxiraneundecanoic acid, 3-pentyl, methyl ester, cis), CID: 5,486,971 (pregabalin), and CID: 131,411(arabidol).

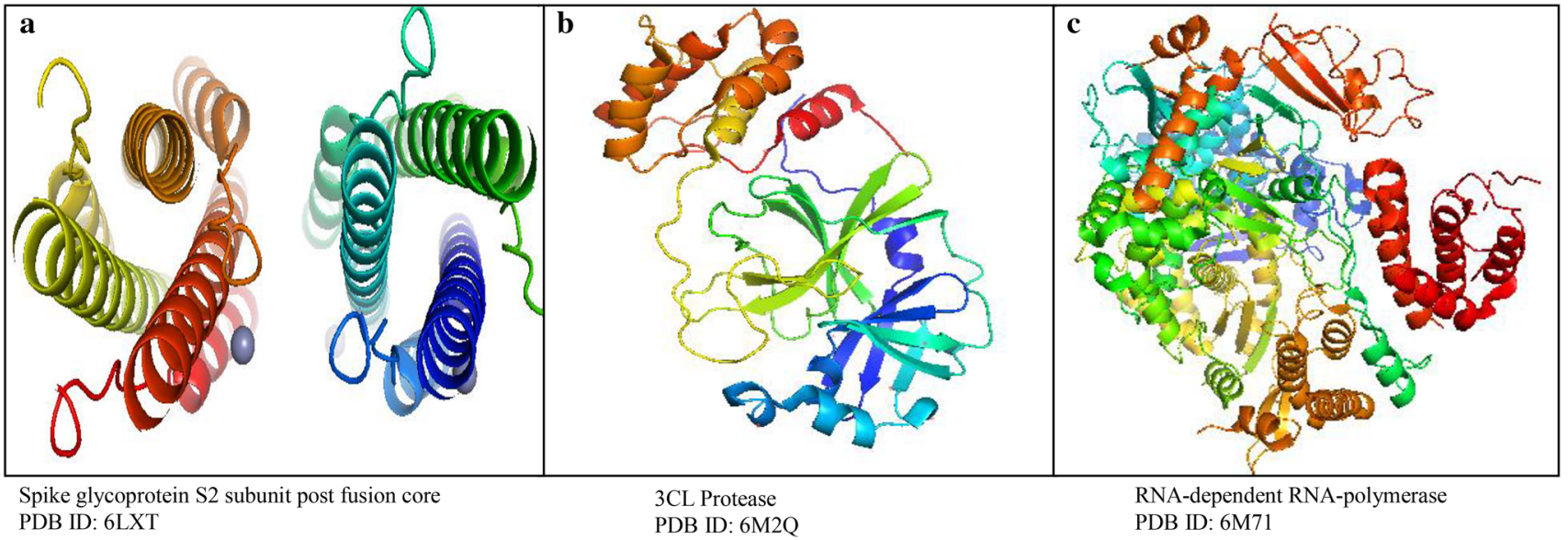

Fig. 1 The 3D ribbon structures representation of SARS-CoV-2 target proteins a spike glycoprotein S2 subunit post-fusion core, b 3CL protease and $\mathbf{c}$ RNA-dependent RNA polymerase visualized using PyMOL 


\subsubsection{Molecular Docking analysis}

Protein-small ligand molecule docking was performed using the PatchDock algorithm (Prabhu and Rajeswari 2016; Yadav et al. 2017; Surana et al. 2018). The parameters were set, the complex type was selected, and clustering root mean square deviation (RMSD) was set to $1.5 \AA$ for protein-ligand interaction. The protein receptor and small ligand molecule were uploaded in PDB format followed by form submission. The following output will be further sent by PatchDock to the given email ID. A 1000 transformed docking candidate generated from PatchDock was refined and re-scored using

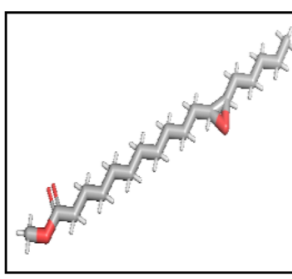

Oxiraneundecanoic acid,3-pentyl,methyl ester, cis CID:71360559

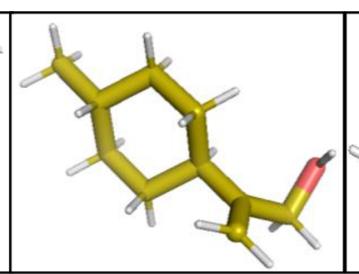

P-menth-8(10)-en-9-ol CID: 543946

(CID: 543946

$\begin{array}{ll}\text { 6H-en-9-ol } & \begin{array}{l}\text { dihydro-2(-methylamino) } \\ \text { dihydrone } 1,7-\end{array} \\ \text { CID: } 135426867\end{array}$

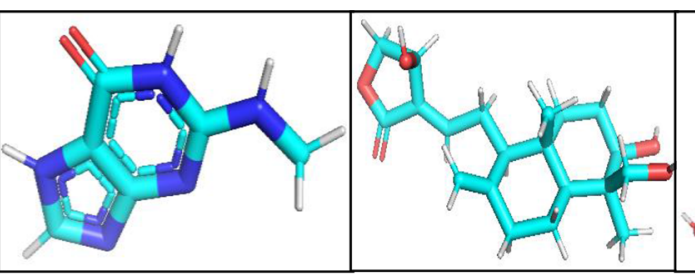

Andrographolide CID: 5318517

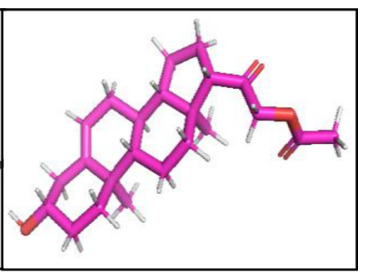

21-acetoxypregenelone

CID: 248856

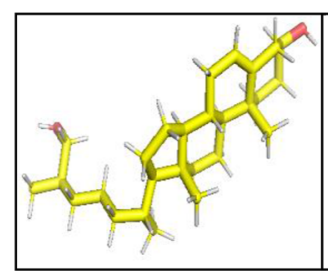

26hydroxycholesterol

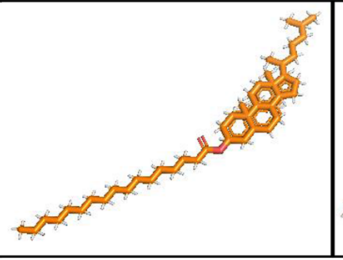

Digitoxin CID: 441207

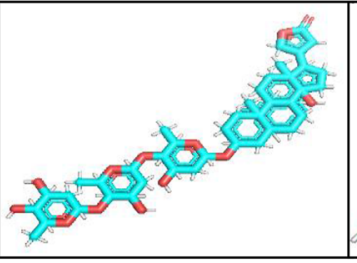

Cholesterol margarate

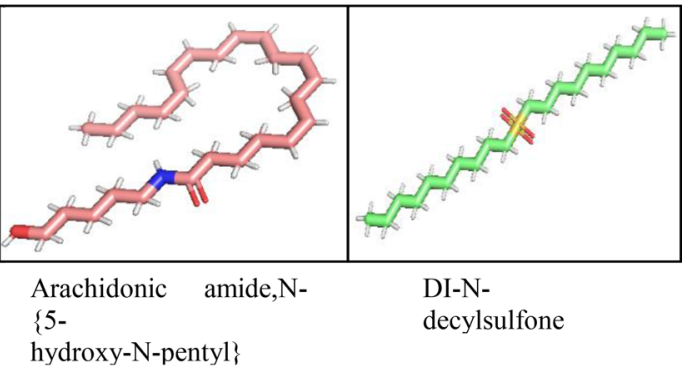

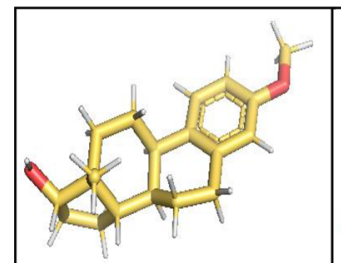

Estra-1,3,5 (10)-trien-

17-beta-ol CID: 13948

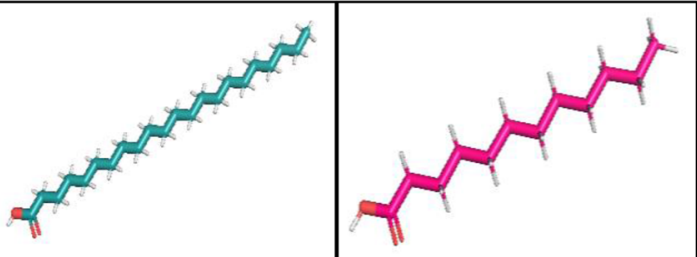

Docosanoic acid CID: 8215

(
14-Dodecanoic CID: 3893

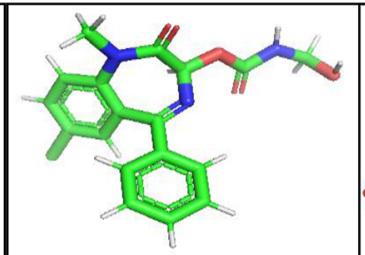

Nor-diazepam, 3-\{Nhydroxymethyl \}amino carbonyloxy CID: 541761

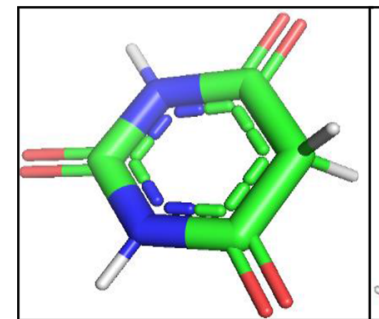

2,4,6- Pyrimidinetrione CID: 6211

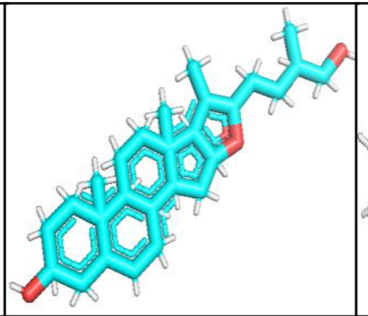

Pseduosarsasapogenin-

5,20-dien

CID: 261799

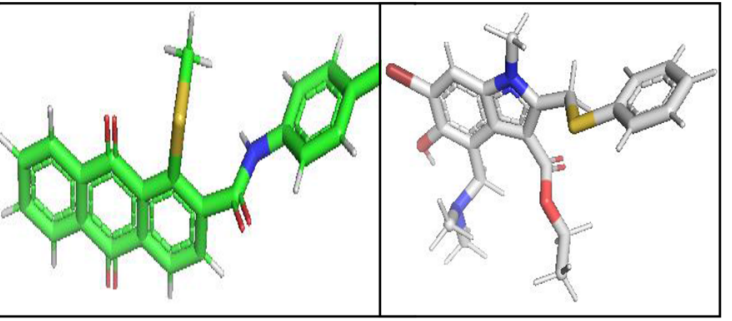

1-methylsulfanyl-9,10dioxo-9,10-dihydroanthracene-2-carbocylic acid CID: 572031

Fig. 2 GC-MS compounds and positive control arabidol ligands downloaded from PubChem data bank with compound ID and visualized using PyMOL for molecular docking 
FireDock. The result was visualized using PyMOL a molecular visualization graphics system tool.

\subsubsection{Drug-likeness analysis of bioactive compounds using SwissADME tool (Daina et al. 2003)}

Chemical structure of compounds was downloaded from PubChem data bank (http://pubchem.ncbi.nlm.nih.gov/) in SDF (structure data format), SwissADME web page was opened, and files were imported from the external file option and were converted into molecular sketcher based on ChemAxon's Marvin JS followed by ADME calculation using default parameters.

\section{Results}

GC-MS analysis reported more than 146 hits and lead compounds from different parts of $P$. maculata (Fig. 3). The compounds identified from acetone leave extracts were more when compared to other solvent extracts. The following hit and lead compounds of $P$. maculata with their bioactivity are listed in Tables 1, 2, and 3.

A molecular docking study performed for nineteen GC-MS compounds using PatchDock with energy minimization and structure refinement using FireDock was analyzed. The targets were docked properly as the binding affinity was shown to be negative and the docked ligand RMSD value was $<2.0 \AA$ (Singh et al. 2017). The binding residues and atomic contact energy of ligands against the target proteins are listed in Table 4. The highest ACE of a docked molecule was considered below $6.00 \mathrm{kcal} / \mathrm{mol}$ (higher negative value); the higher the binding affinity value, the higher the binding potential between molecules. The different GC-MS compounds were interacted with numbers of residues on both side chain and backbone of the target protein as shown in Figs. 4, 5, and 6. A non-covalent (polar) interaction was observed between the docking molecules at a closer distance of $1.5 \AA$. The docking complex showed zero or one-tothree hydrogen bonding between them but formed a specific

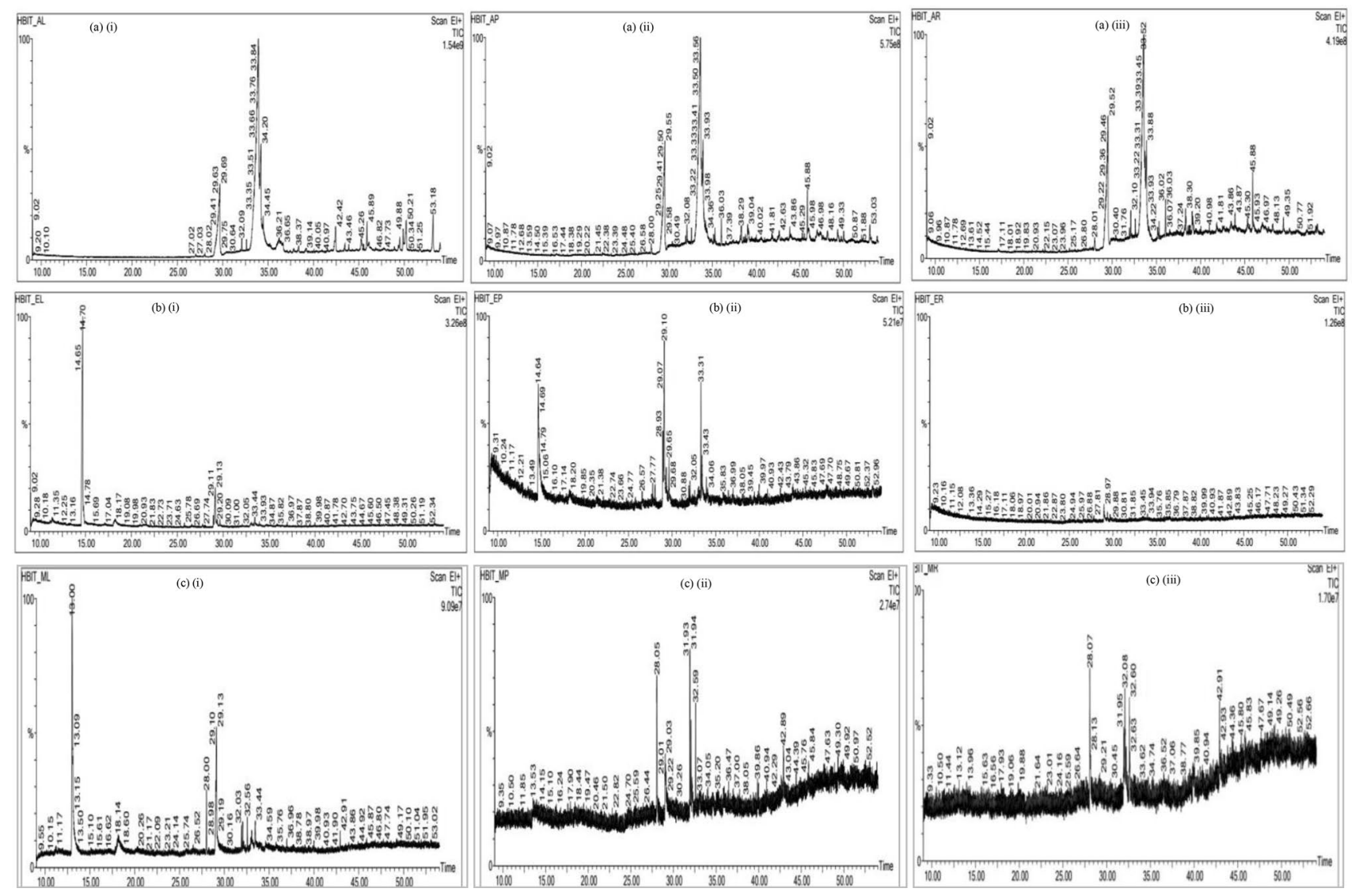

Fig. 3 GC-MS spectrograms showing peaks of compounds of Pleione maculata extracts (a) acetone, (b) ethanol (c) methanol extracts of (i) leave (ii) stem and (iii) root 
Table 1 GC-MS analysis of acetone extracts of Pleione maculata

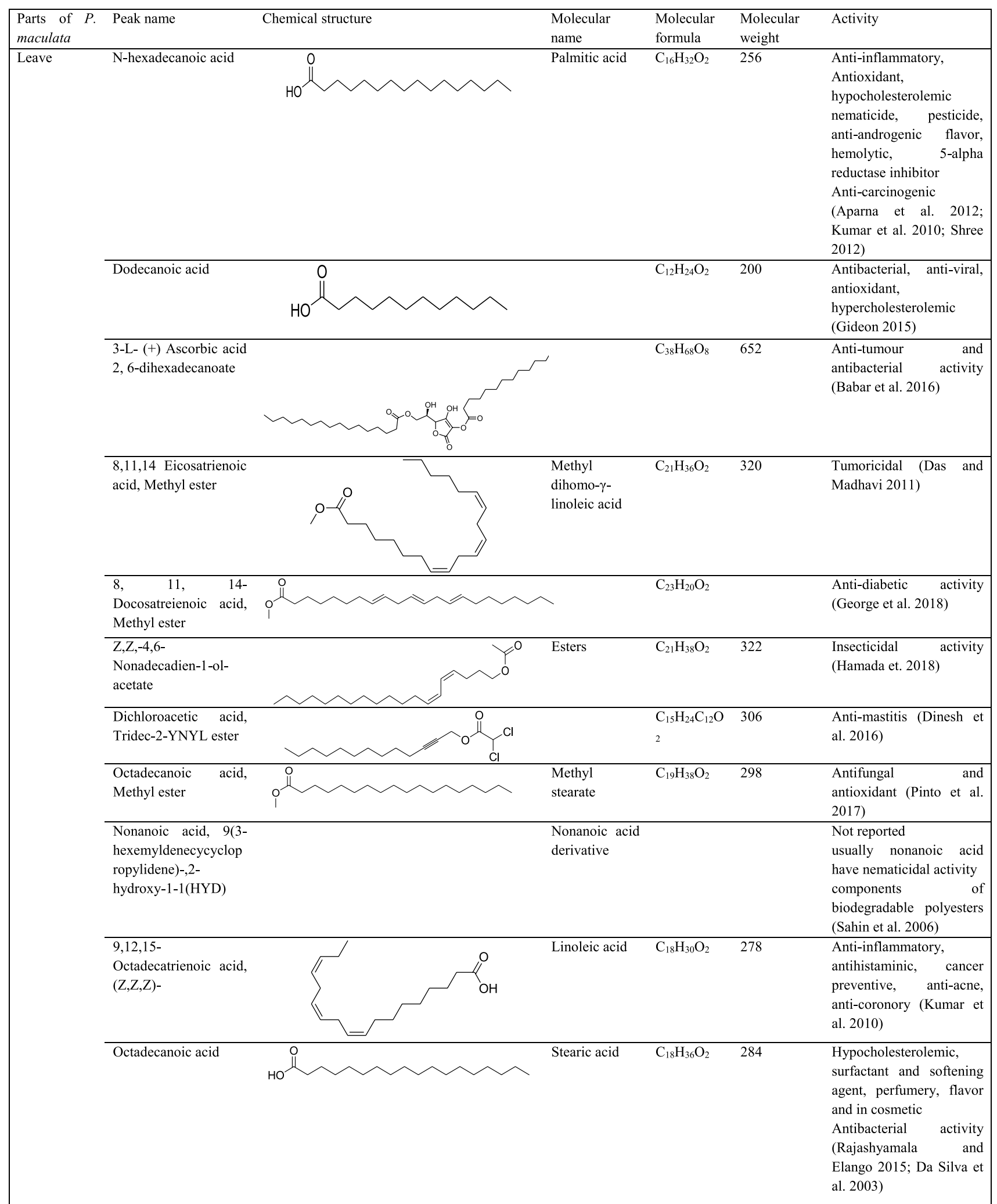


Table 1 (continued)

\begin{tabular}{|c|c|c|c|c|}
\hline $\begin{array}{l}\text { Docosanoic acid/ } \\
\text { Behenic acid }\end{array}$ & Fatty acid & $\mathrm{C}_{23} \mathrm{SH}_{44} \mathrm{O}_{2}$ & 340 & $\begin{array}{l}\text { Anti-cancer potential } \\
\text { against MCF7 and HeLa } \\
\text { cell lines, } \\
\text { Hair moisturizer (Lawal } \\
\text { et al. 2015; Eswaraiah et } \\
\text { al. 2020) }\end{array}$ \\
\hline $\begin{array}{l}\text { Alpha-Ketostearic } \\
\text { acid, ethyl ester }\end{array}$ & & $\mathrm{C}_{20} \mathrm{H}_{38} \mathrm{O}_{3}$ & 326 & No activity reported \\
\hline $\begin{array}{l}\text { Cyclopropaneoctanoic } \\
\text { acid, 2-[[2- } \\
\text { Ethylcyclopropyl)Met } \\
\text { hyl]cyclo }\end{array}$ & & $\mathrm{C}_{22} \mathrm{H}_{38} \mathrm{O}_{2}$ & 334 & $\begin{array}{l}\text { Anti-carcinogenic (Shree } \\
\text { 2012) }\end{array}$ \\
\hline $\begin{array}{l}1- \\
\text { naphthaleneproponal, } \\
\text { alpha-ethyl } \\
\text { Decahydro-5- } \\
\text { (hydroxymethyl)- }\end{array}$ & & $\mathrm{C}_{20} \mathrm{H}_{36} \mathrm{O}_{2}$ & 308 & No activity reported \\
\hline Terpin hydrate & & $\mathrm{C}_{10} \mathrm{H}_{22} \mathrm{O}_{3}$ & 172 & $\begin{array}{l}\text { Expectorant commonly } \\
\text { used to loosen mucus in } \\
\text { patients presenting with } \\
\text { acute or chronic } \\
\text { bronchitis and related } \\
\text { conditions (Jahan et al. } \\
\text { 2015) }\end{array}$ \\
\hline $\begin{array}{l}\text { Cis, Cis, Cis-7,10,13- } \\
\text { Hexadecatrienal }\end{array}$ & aldehyde & $\mathrm{C}_{16} \mathrm{H}_{26} \mathrm{O}$ & 234 & $\begin{array}{l}\text { No activity reported } \\
\text { (Prabhadevi et al. 2012; } \\
\text { Abulaziz et al. 2019) }\end{array}$ \\
\hline Cholest-4-en-3-one & Cholestenone & $\mathrm{C}_{27} \mathrm{H}_{44} \mathrm{O}$ & 384 & $\begin{array}{l}\text { Anti-obesity, suppress } \\
\text { body weight and body fat } \\
\text { accumulation (Suzuki } \\
1993,1998 \text { ) }\end{array}$ \\
\hline Diazoprogesterone & $\begin{array}{l}\text { Steroid, } \\
\text { Nitrogen } \\
\text { compound }\end{array}$ & $\mathrm{C}_{21} \mathrm{H}_{30} \mathrm{~N}_{4}$ & 338 & $\begin{array}{l}\text { Antimicrobial } \\
\text { anti-inflammatory, } \\
\text { Hepatoprotective, } \\
\text { Duiretic, Anti-cancer } \\
\text { Anti-HIV (Gopinath et al. } \\
\text { 2013; Jothi et al. 2015) }\end{array}$ \\
\hline $\begin{array}{l}\text { 26-hydroxy } \\
\text { cholesterol }\end{array}$ & $\begin{array}{l}\text { steroid } \\
\text { compound }\end{array}$ & $\mathrm{C}_{27} \mathrm{H}_{46} \mathrm{O}_{2}$ & 402 & $\begin{array}{l}\text { Biomarkers in diagnosis } \\
\text { of Alzheimers disease } \\
(\mathrm{AD}) \text { and other } \\
\text { neurodegenerative } \\
\text { disorders (De Knock } \\
2016)\end{array}$ \\
\hline $\begin{array}{l}\text { pregn-4-ene-1,20- } \\
\text { Dione, } \quad 12 \text {-hydroxy- } \\
\text { 16,17-dimethyl- }\end{array}$ & $\begin{array}{l}\text { Steroid } \\
\text { compound }\end{array}$ & $\mathrm{C}_{23} \mathrm{H}_{34} \mathrm{O}_{3}$ & 358 & $\begin{array}{l}\text { Sexual disorders, } \\
\text { baldness, anti-psioriatic, } \\
\text { anti-pyretic, anti-allergic } \\
\text { (Ansarali et al. 2018) }\end{array}$ \\
\hline
\end{tabular}


Table 1 (continued)

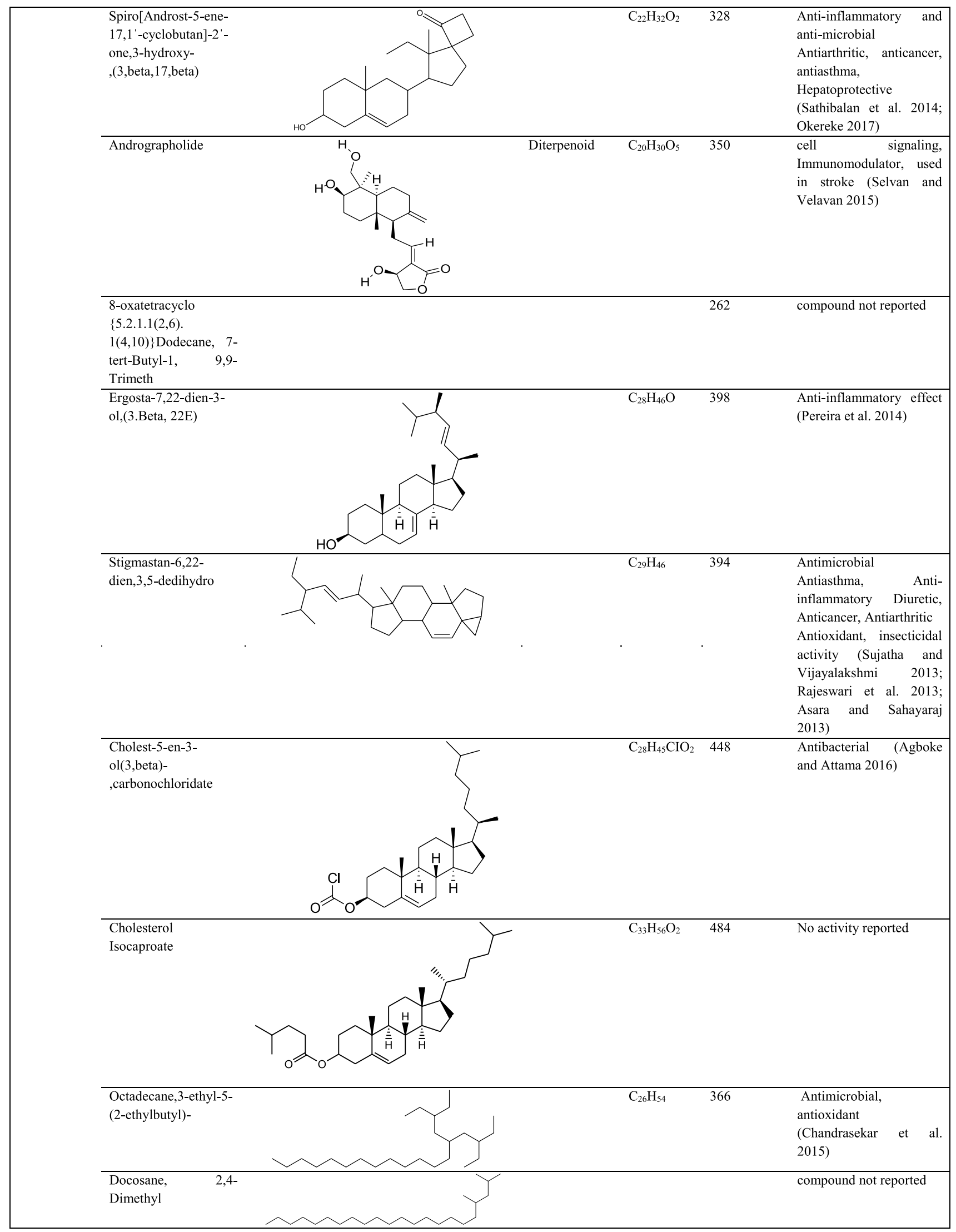


Table 1 (continued)

\begin{tabular}{|c|c|c|c|c|c|}
\hline & DL-alpha-tocopherol & Vitamin E & $\mathrm{C}_{29} \mathrm{H}_{50} \mathrm{O}_{2}$ & 430 & $\begin{array}{l}\text { Anti-oxidation effects, } \\
\text { protects human skin } \\
\text { against cytotoxic effect of } \\
\text { UVB and dietary } \\
\text { supplement (Kondo et al. } \\
\text { 1990; Jialal and Grundy } \\
\text { 1992) }\end{array}$ \\
\hline & Vitamin E & Vitamin E & $\mathrm{C}_{29} \mathrm{H}_{50} \mathrm{O}_{2}$ & 430 & $\begin{array}{l}\text { Analgesic, anti-cataract, } \\
\text { anti-coronary, anti- } \\
\text { diabetic, antioxidant, } \\
\text { Hepatoprotective, } \\
\text { Vasodilator, protein- } \\
\text { kinase-c-inhibitor, } \\
\text { anticancer, Lipoxygenase } \\
\text { inhibitor, Anti-bronchitic, } \\
\text { anti-coronary (Jothi et al. } \\
\text { 2015; Rajalakshmi and } \\
\text { Mohan 2016) }\end{array}$ \\
\hline & $\begin{array}{l}\text { 6h-Purin-6-one,1,7- } \\
\text { dihydro- } \\
\text { 2(methylamino) }\end{array}$ & $\begin{array}{l}\text { imidazole } \\
\text { derivatives }\end{array}$ & $\mathrm{C}_{7} \mathrm{H}_{8} \mathrm{~N}_{40}$ & 164 & $\begin{array}{l}\text { Acts as anti-viral (active } \\
\text { against HSV-1 and HSV- } \\
\text { 2) (Kumar et al 2017) }\end{array}$ \\
\hline & $\begin{array}{l}\text { 1H-Purin-2-amine-6- } \\
\text { methoxy }\end{array}$ & & $\mathrm{C}_{6} \mathrm{H}_{7} \mathrm{~N}_{50}$ & 165 & No activity reported \\
\hline & $\begin{array}{l}\text { 26-nor-5-cholesten- } \\
\text { 3,beta,-ol-25-one }\end{array}$ & & $\mathrm{C}_{26} \mathrm{H}_{42} \mathrm{O}_{2}$ & 386 & $\begin{array}{l}\text { Anti-tumor, anti- } \\
\text { inflammatory, anti- } \\
\text { oxidant and anti-bacterial } \\
\text { (Yuvaraj and Arul 2014) }\end{array}$ \\
\hline & $\begin{array}{l}21- \\
\text { Acetoxypregnenolone }\end{array}$ & & $\mathrm{C}_{25} \mathrm{H}_{36} \mathrm{O}_{5}$ & 416 & $\begin{array}{l}\text { Anti-microbial, } \\
\text { antioxidant, targets } \\
\text { antisense strands (targets } \\
\text { single stranded RNA } \\
\text { complimentary to protein } \\
\text { coding mRNA which } \\
\text { hybridizes and block } \\
\text { translation of protein) } \\
\text { mainly use in gene } \\
\text { knockdown } \\
\text { Antiproliferative activity } \\
\text { against melanoma cells } \\
\text { (Chidambaram et al. } \\
\text { 1996; Kim et al. 2009; } \\
\text { Chen et al. 2014) }\end{array}$ \\
\hline Stem & $\begin{array}{l}\text { Hexadecanoic acid, } \\
\text { methyl ester }\end{array}$ & & $\mathrm{C}_{17} \mathrm{H}_{34} \mathrm{O}_{2}$ & 270 & $\begin{array}{l}\text { Antioxidant, } \\
\text { hypocholesterolenic, } \\
\text { flavor, hemolytic 5-alpha } \\
\text { reductase inhibitor, } \\
\text { nematicide, } \\
\text { antiandrogenic (Easwaran } \\
\text { and Ramani 2014) }\end{array}$ \\
\hline & N-hexadecanoic acid & Palmitic acid & $\mathrm{C}_{16} \mathrm{H}_{32} \mathrm{O}_{2}$ & 256 & $\begin{array}{l}\text { Anti-inflammatory, } \\
\text { Antioxidant, } \\
\text { hypocholesterolemic } \\
\text { nematicide, pesticide, } \\
\text { anti-androgenic flavor, } \\
\text { hemolytic, 5-alpha } \\
\text { reductase inhibitor } \\
\text { (Kumar et al. 2010; } \\
\text { Aparna et al. 2012) } \\
\end{array}$ \\
\hline & Isopropyl palmitate & & $\mathrm{C}_{19} \mathrm{H}_{38} \mathrm{O}_{2}$ & 298 & $\begin{array}{l}\text { Anti-proliferative activity } \\
\text { (Saxena et al. 2007) }\end{array}$ \\
\hline
\end{tabular}


Table 1 (continued)

\begin{tabular}{|c|c|c|c|c|}
\hline $\begin{array}{l}\text { 8,11,14 Eicosatrienoic } \\
\text { acid, methyl ester }\end{array}$ & $\begin{array}{l}\text { Methyl } \\
\text { dihomo- } \gamma- \\
\text { linoleic acid }\end{array}$ & $\mathrm{C}_{21} \mathrm{H}_{36} \mathrm{O}_{2}$ & 320 & $\begin{array}{l}\text { Tumoricidal (Das and } \\
\text { Madhavi 2011) }\end{array}$ \\
\hline $\begin{array}{l}\text { 10-undecynoic acid, } \\
\text { methyl ester }\end{array}$ & & $\mathrm{C}_{12} \mathrm{H}_{20} \mathrm{O}_{2}$ & 196 & $\begin{array}{l}\text { Anti-oxidant (Palakkal et } \\
\text { al. 2017) }\end{array}$ \\
\hline $\begin{array}{l}\text { Octadecanoic acid, } \\
\text { methyl ester }\end{array}$ & $\begin{array}{l}\text { Methyl } \\
\text { stearate }\end{array}$ & $\mathrm{C}_{19} \mathrm{H}_{38} \mathrm{O}_{2}$ & 298 & $\begin{array}{l}\text { Antifungal and } \\
\text { antioxidant (Pinto et al. } \\
\text { 2017) }\end{array}$ \\
\hline $\begin{array}{l}9,12 \text {-octadecadienoyl } \\
\text { chloride, (Z,Z)- }\end{array}$ & $\begin{array}{l}\text { Linoleoyl } \\
\text { chloride }\end{array}$ & $\mathrm{C}_{18} \mathrm{H}_{31} \mathrm{ClO}$ & 298 & $\begin{array}{l}\text { Anticancer, Anticoronary, } \\
\text { Antieczemic, } \\
\text { Antihistamic (Kumar et } \\
\text { al. 2015) }\end{array}$ \\
\hline Octadecanoic acid & Stearic acid & $\mathrm{C}_{18} \mathrm{H}_{36} \mathrm{O}_{2}$ & 284 & $\begin{array}{l}\text { Hypocholesterolemic, } \\
\text { surfactant and softening } \\
\text { agent, perfumery, flavor } \\
\text { and in cosmetic } \\
\text { Antibacterial activity (Da } \\
\text { Silva et al. 2003; } \\
\text { Rajashyamala and Elango } \\
2015 \text { ) }\end{array}$ \\
\hline $\begin{array}{l}\text { Hexadecanoic acid, 1- } \\
\text { (hydroxymethyl)-1,2- } \\
\text { ethanediyl ester }\end{array}$ & $\begin{array}{l}\text { Glyceryl } 1,2- \\
\text { dipalmitate }\end{array}$ & $\mathrm{C}_{35} \mathrm{H}_{68} \mathrm{O}_{5}$ & 568 & $\begin{array}{l}\text { Antimicrobial, } \\
\text { Antioxidant (Kumar et al } \\
\text { 2013) }\end{array}$ \\
\hline $\begin{array}{l}\text { 2-piperidinone,N-(4- } \\
\text { Bromo-N-butyl)- }\end{array}$ & Alkaloid & $\mathrm{C}_{9} \mathrm{H}_{34} \mathrm{O}_{7} \mathrm{~S}$ & 370 & $\begin{array}{l}\text { Anti-inflammatory, anti- } \\
\text { microbial, anti-cancer } \\
\text { (Jothi et al. 2015) }\end{array}$ \\
\hline oleic acid & $\begin{array}{l}\text { Carboxylic } \\
\text { acid }\end{array}$ & $\mathrm{C}_{18} \mathrm{H}_{34} \mathrm{O}_{2}$ & 282.468 & $\begin{array}{l}\text { Anti-inflammatory, } \\
\text { cancer preventive, } \\
\text { antimicrobial, anti- } \\
\text { tumour activity (Padma et } \\
\text { al. 2018; Karunanithi and } \\
\text { Venkatachalam 2019) }\end{array}$ \\
\hline $\begin{array}{l}\text { Hexadecanoic acid, 3- } \\
\text { [(trimethylsilyl)oxy]pr } \\
\text { opyl ester }\end{array}$ & $\begin{array}{l}\text { 3- } \\
\text { Trimethylsilyl } \\
\text { oxypropyl } \\
\text { hexadecanoate }\end{array}$ & $\mathrm{C}_{22} \mathrm{H}_{46} \mathrm{O}_{3} \mathrm{Si}$ & 386 & $\begin{array}{l}\text { Antimicrobial, } \\
\text { Antioxidant } \\
\text { (Chandrasekar et al. } \\
\text { 2015) }\end{array}$ \\
\hline $\begin{array}{l}\text { 4-cyanobenzoic acid, } \\
\text { Tridec-2-YNYL,ester }\end{array}$ & & $\mathrm{C}_{21} \mathrm{H}_{27} \mathrm{NO}_{2}$ & 325 & No activity reported \\
\hline $\begin{array}{l}\text { Bisnor-7-desoxycholic } \\
\text { acid }\end{array}$ & & $\mathrm{C}_{22} \mathrm{H}_{36} \mathrm{O}_{4}$ & 364 & $\begin{array}{l}\text { Antimicrobial, } \\
\text { Antioxidant, Anticancer } \\
\text { (Wei et al. 2011) }\end{array}$ \\
\hline 26-hydroxycholesterol & LDL & $\mathrm{C}_{27} \mathrm{H}_{46} \mathrm{O}_{2}$ & 402 & $\begin{array}{l}\text { Inhibition of cholesterol } \\
\text { synthesis (Javitt 1990) }\end{array}$ \\
\hline
\end{tabular}


Table 1 (continued)

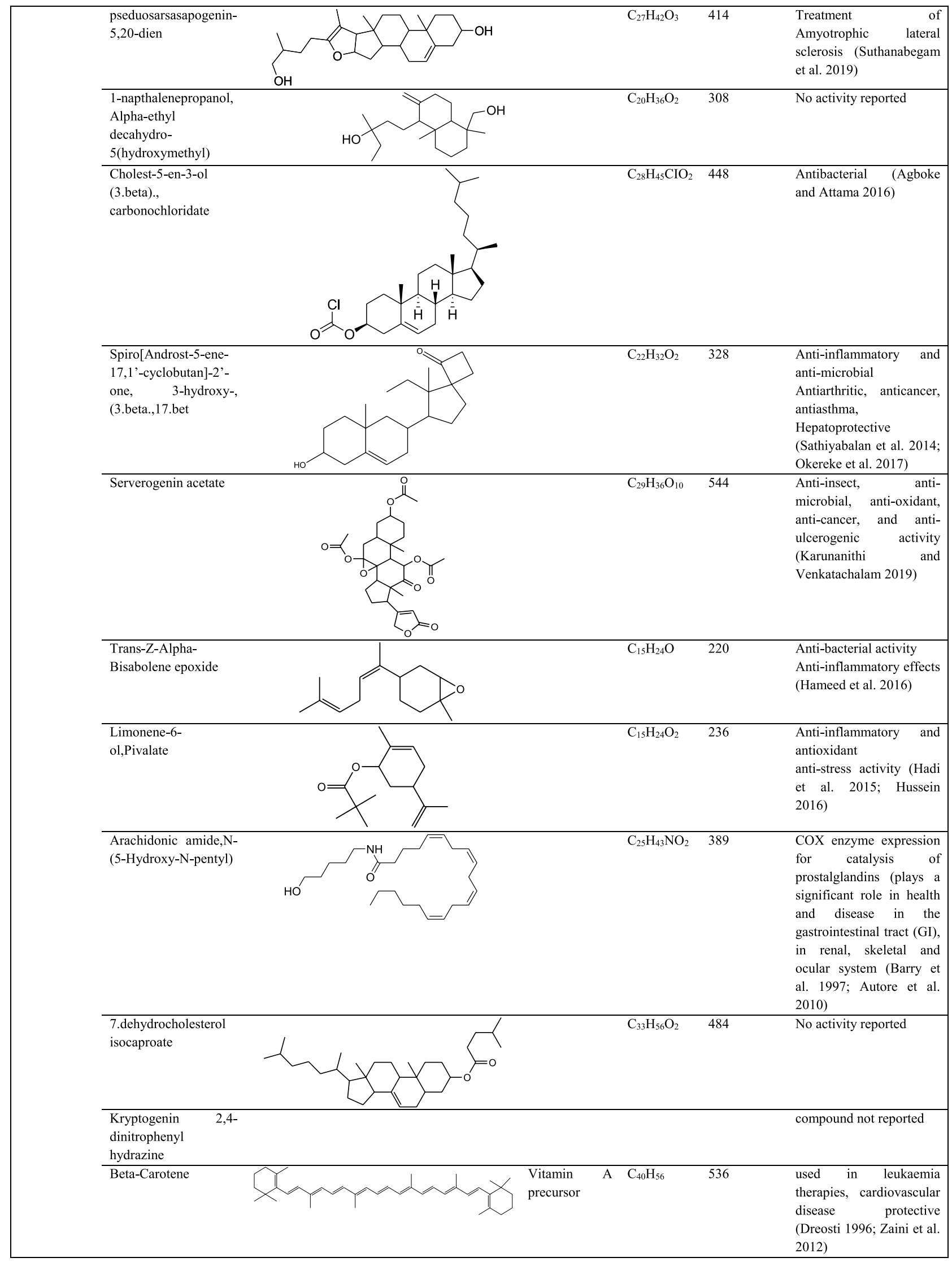


Table 1 (continued)

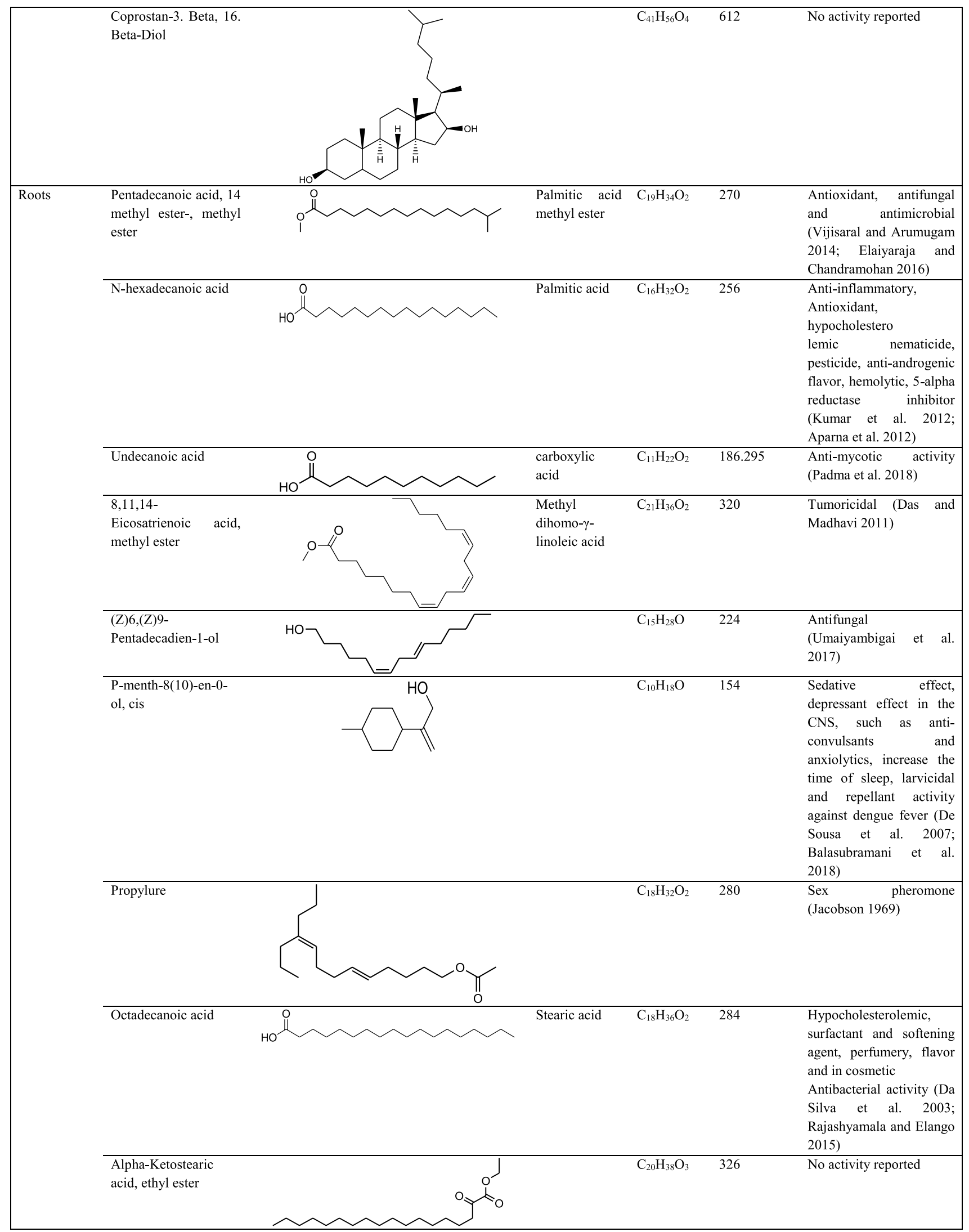


Table 1 (continued)

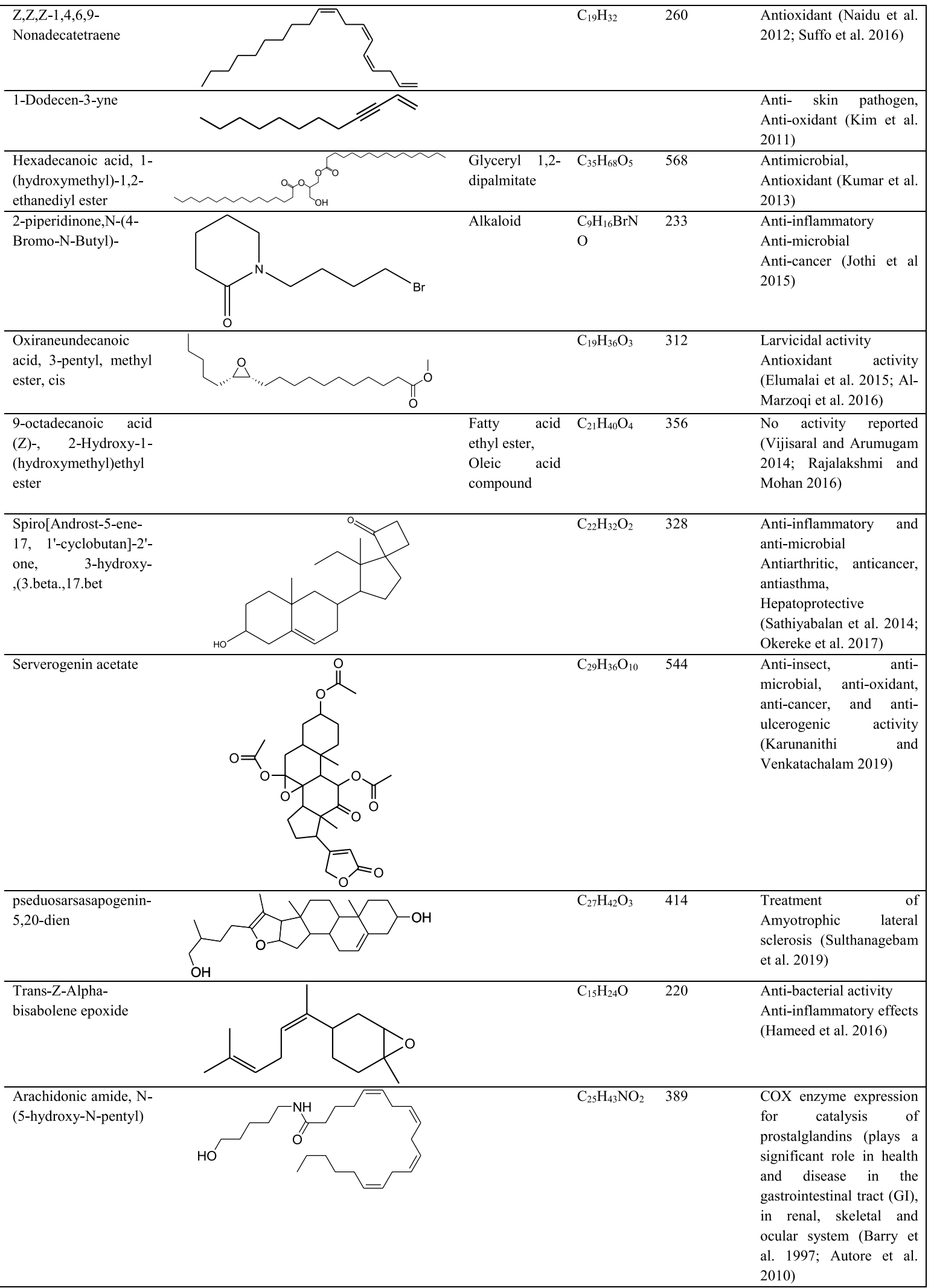


Table 1 (continued)

\begin{tabular}{|c|c|c|c|c|}
\hline Stigmastan-3,5-diene & & $\mathrm{C}_{29} \mathrm{H}_{48}$ & 396 & $\begin{array}{l}\text { Antimicrobial and } \\
\text { Antioxidant (Khan et al. } \\
\text { 2016) }\end{array}$ \\
\hline Beta-Sitosterol acetate & & $\mathrm{C}_{31} \mathrm{H}_{52} \mathrm{O}_{2}$ & 456 & $\begin{array}{l}\text { Anti-inflammatory, } \\
\text { inducing apoptosis, } \\
\text { chemoprotective or } \\
\text { chemoprotective effects, } \\
\text { angiogenic effect, } \\
\text { prostatic cancer treatment } \\
\text { (Saeidmia et al. 2014) }\end{array}$ \\
\hline Digitoxin & $\begin{array}{l}\text { cardiac } \\
\text { glycoside }\end{array}$ & $\mathrm{C}_{41} \mathrm{H}_{64} \mathrm{O}_{13}$ & 764 & $\begin{array}{l}\text { used for chronic cardiac } \\
\text { insufficiency (fatigue, } \\
\text { shortness of breath and } \\
\text { edema) (Vardanyan and } \\
\text { Hruby 2006) }\end{array}$ \\
\hline $\begin{array}{l}\text { Ergosta-4,6,22-Trien- } \\
\text { 3, alpha-ol }\end{array}$ & steroid & $\mathrm{C}_{28} \mathrm{H}_{44} \mathrm{O}$ & 396 & $\begin{array}{l}\text { Anti-microbial, anti- } \\
\text { inflammatory, anti- } \\
\text { cancer, anti-arthritic, anti- } \\
\text { asthma, diuretic (Lalithi } \\
\text { et al. 2015) }\end{array}$ \\
\hline Ascorbyl palmitate & $\begin{array}{l}\text { Ascorbic acid } \\
\text { ester }\end{array}$ & $\mathrm{C}_{22} \mathrm{H}_{38} \mathrm{O}_{7}$ & 414 & $\begin{array}{l}\text { Food additive and } \\
\text { cosmetic ingredient } \\
\text { (Tuffino et al. 2019) }\end{array}$ \\
\hline $\begin{array}{l}\text { D-mannitol, } \\
\text { Decylsulfonyl }\end{array}$ & $\begin{array}{l}\text { Sulfur } \\
\text { compound }\end{array}$ & $\mathrm{C}_{16} \mathrm{H}_{34} \mathrm{O}_{7} \mathrm{~S}$ & 370 & $\begin{array}{l}\text { antimicrobial } \\
\text { antidiabetic } \\
\text { (Muthukrishnan and } \\
\text { Thinakaran 2012; } \\
\text { Alagammal et al. 2012; } \\
\text { Jothi et al. 2015; Ezekiel } \\
\text { et al. 2018) }\end{array}$ \\
\hline DI-N-Decylsulfone & & $\mathrm{C}_{20} \mathrm{H}_{42} \mathrm{O}_{2} \mathrm{~S}$ & 346 & $\begin{array}{l}\text { Anti-microbial and anti- } \\
\text { cancer } \\
\text { Larvicidal activity } \\
\text { (Vijayakumari and Raj } \\
\text { 2019; Karthi et al. 2020) }\end{array}$ \\
\hline $\begin{array}{l}\text { Alpha-L- } \\
\text { Fucopyranose 1,2.3,4- } \\
\text { Bis (Benzeneboonate) } \\
\end{array}$ & & $\mathrm{C}_{18} \mathrm{H}_{18} \mathrm{~B}_{2} \mathrm{O}_{5}$ & 336 & No activity reported \\
\hline cholesterol margarate & & $\mathrm{C}_{44} \mathrm{H}_{78} \mathrm{O}_{2}$ & 639 & No activity reported \\
\hline $\begin{array}{l}\text { N-decyl-Alpha,D-2- } \\
\text { Deoxyglycoside }\end{array}$ & & & & Compound not reported \\
\hline
\end{tabular}


Table 2 GC-MS analysis of ethanol extracts of Pleione maculata

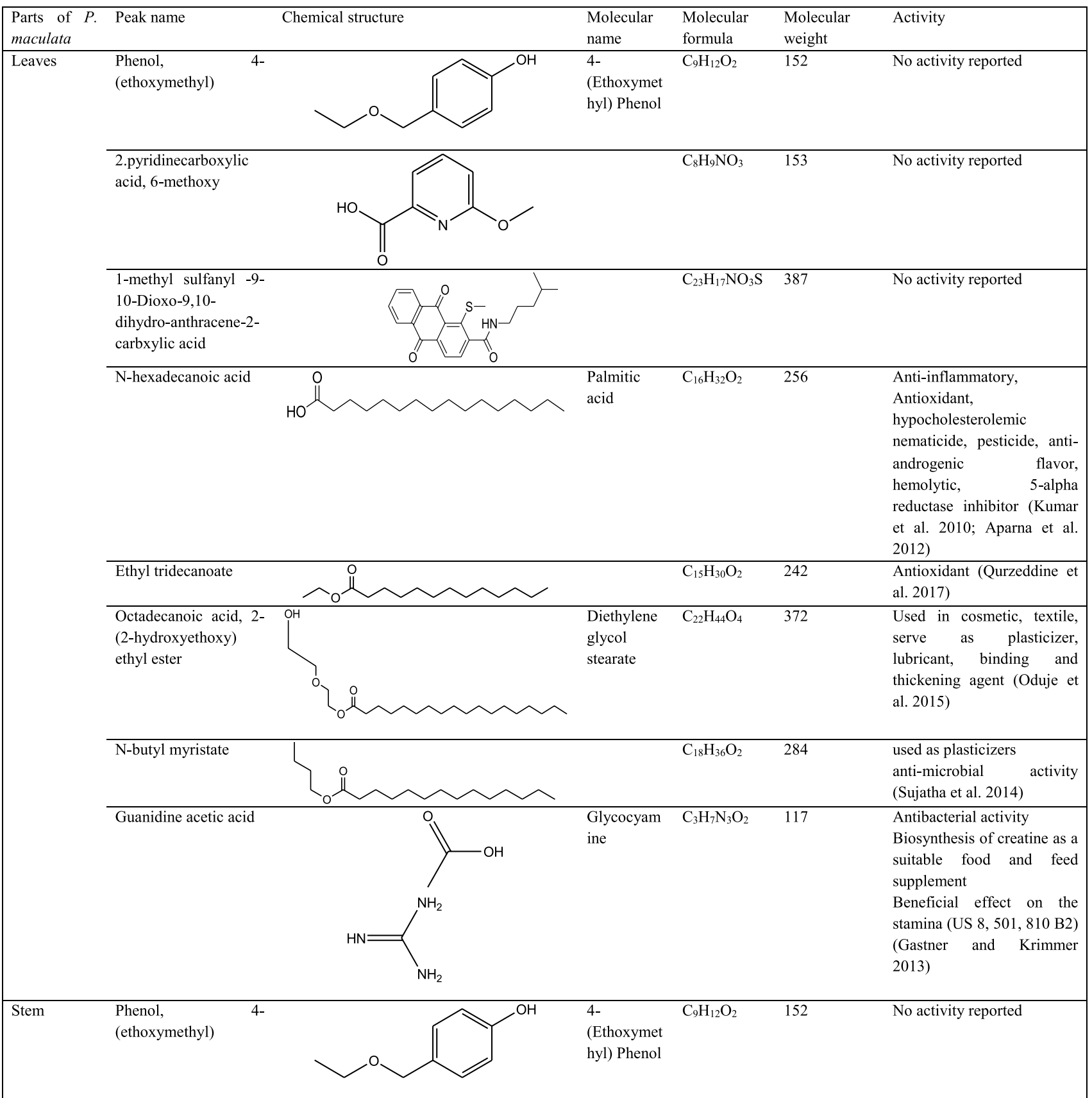


Table 2 (continued)

\begin{tabular}{|c|c|c|c|c|}
\hline $\begin{array}{l}1,2- \\
\text { benzenedicarboxylic } \\
\text { acid, butyl octyl ester }\end{array}$ & $\begin{array}{l}\text { Phthalic } \\
\text { acid, butyl } \\
\text { octyl ester, } \\
\text { Plasticizer } \\
\text { BOP }\end{array}$ & $\mathrm{C}_{20} \mathrm{H}_{30} \mathrm{O}_{4}$ & 334 & $\begin{array}{l}\text { Antimicrobial, Antifouling } \\
\text { (Lakshmi and Rajalakshmi } \\
\text { 2011; Khalil et al. 2014) }\end{array}$ \\
\hline N-hexadecanoic acid & $\begin{array}{l}\text { Palmitic } \\
\text { acid }\end{array}$ & $\mathrm{C}_{16} \mathrm{H}_{32} \mathrm{O}_{2}$ & 256 & $\begin{array}{l}\text { Anti-inflammatory, } \\
\text { Antioxidant, } \\
\text { hypocholesterolemic } \\
\text { nematicide, pesticide, anti- } \\
\text { androgenic } \\
\text { hemolytic, flavor, } \\
\text { reductase inhibitor (Kumar } \\
\text { et al. 2010; Aparna et al. } \\
2012 \text { ) }\end{array}$ \\
\hline $\begin{array}{l}\text { Hexadecanoic acid, } \\
\text { ethyl ester }\end{array}$ & $\begin{array}{l}\text { Palmitic } \\
\text { acid ester }\end{array}$ & $\mathrm{C}_{18} \mathrm{H}_{36} \mathrm{O}_{2}$ & 284 & $\begin{array}{lr}\text { Antioxidant, } & \text { Hemolytic, } \\
\text { hypocholesterone, } & \text { Flavor, } \\
\text { Nematicide, } & \text { Anti- } \\
\text { androgenic (Tyagi and } \\
\text { Agarwal 2016) }\end{array}$ \\
\hline $\begin{array}{l}\text { 9,12-octadecadienoic } \\
\text { acid }(\mathrm{Z}, \mathrm{Z}) \text { - }\end{array}$ & $\begin{array}{l}\text { Conjugated } \\
\text { Linoleic } \\
\text { acid }\end{array}$ & $\mathrm{C}_{18} \mathrm{H}_{32} \mathrm{O}_{2}$ & 280 & $\begin{array}{l}\text { Anti-Inflammatory, } \\
\text { hypocholesterolic, cancer } \\
\text { preventive, } \\
\text { Hepatoprotective, } \\
\text { nematicide, antihistaminic, } \\
\text { antieczemic, antiacne, 5- } \alpha \\
\text { reductase inhibitor, anti- } \\
\text { coronary, antimicrobial } \\
\text { (Adeoye-Isijola et al. 2018) }\end{array}$ \\
\hline $\begin{array}{l}\text { Linoleic acid ethyl } \\
\text { ester }\end{array}$ & & $\mathrm{C}_{20} \mathrm{H}_{34} \mathrm{O}_{2}$ & 308 & $\begin{array}{l}\text { Hypocholesterolemic, } \\
\text { nematicide, anti-arthritic, } \\
\text { hepatoproctective, anti- } \\
\text { androgenic, } \\
\text { hypycholesterolemic, 5- } \\
\text { alpha reductase inhibitor, } \\
\text { anti-histaminic, anti- } \\
\text { coronary, insectifuge, anti- } \\
\text { eczemic, anti-acne } \\
\text { (Chidambarampillai and } \\
\text { Mohan 2013; Tyagi and } \\
\text { Agarwal 2016) }\end{array}$ \\
\hline Isopropyl linoleate & & $\mathrm{C}_{21} \mathrm{H}_{38} \mathrm{O}_{2}$ & 322 & $\begin{array}{l}\text { Antioxidant, antidiabetic, } \\
\text { anti-inflammatory } \\
\text { Formulation of skin and hair } \\
\text { care products, facial makeup } \\
\text { (Rajendra et al. 2017; } \\
\text { Rautela et al. 2018) }\end{array}$ \\
\hline $\begin{array}{l}\text { Dichloroacetic acid, } \\
\text { dodec-9-YNYL }\end{array}$ & & $\mathrm{C}_{14} \mathrm{H}_{23} \mathrm{ClO}_{2}$ & 292 & $\begin{array}{l}\text { Acidifier, arachidonic acid } \\
\text { inhibitor, increase aromatic } \\
\text { amino acid decarboxylase } \\
\text { activity, inhibit production } \\
\text { of uric acid (Mohammad et } \\
\text { al. 2019) }\end{array}$ \\
\hline Oleic acid & & $\mathrm{C}_{18} \mathrm{H}_{34} \mathrm{O}_{2}$ & 282 & $\begin{array}{lr}\text { Antibacterial } & \text { activity } \\
\text { (Abubakar and Majinda } \\
\text { 2016) }\end{array}$ \\
\hline $\begin{array}{l}\text { Estra-1,3,5 (10)-Trien- } \\
\text { 17-Beta-ol }\end{array}$ & & $\mathrm{C}_{18} \mathrm{H}_{24} \mathrm{O}$ & 256 & $\begin{array}{ll}\text { Anti-arrhythmic } & \text { activity } \\
\text { (Al-Gara'wi 2019) } & \end{array}$ \\
\hline
\end{tabular}


Table 2 (continued)

\begin{tabular}{|c|c|c|c|c|c|}
\hline & $\begin{array}{l}\text { 9-Oxononanoic acid } \\
\text { (9-ONA) }\end{array}$ & & $\mathrm{C}_{9} \mathrm{H}_{16} \mathrm{O}_{3}$ & 172 & $\begin{array}{l}\text { Lipid peroxidation (treats } \\
\text { disorder such as } \\
\text { inflammation, } \\
\text { atherosclerosis and other } \\
\text { degenerative diseases) } \\
\text { induces phospholipase A2 } \\
\text { activity and thromboxane } \\
\text { A2 production in human } \\
\text { blood (Ren et al. 2013) }\end{array}$ \\
\hline \multirow[t]{3}{*}{ Roots } & $\begin{array}{l}\text { Phthalic acid, butyl } \\
\text { hexyl ester }\end{array}$ & $\begin{array}{l}\text { Butyl hexyl } \\
\text { phthalate }\end{array}$ & $\mathrm{C}_{18} \mathrm{H}_{26} \mathrm{O}_{4}$ & 306 & Antimicrobial (Ingole 2016) \\
\hline & $\begin{array}{l}1,2- \\
\text { benzenedicarboxylic } \\
\text { acid, butyl octyl ester }\end{array}$ & $\begin{array}{l}\text { Phthalic } \\
\text { acid, butyl } \\
\text { octyl ester, }\end{array}$ & $\mathrm{C}_{20} \mathrm{H}_{30} \mathrm{O}_{4}$ & 334 & $\begin{array}{l}\text { Antimicrobial, Antifouling } \\
\text { (Lakshmi and Rajalakshmi } \\
\text { 2011; Khalil et al. 2014) }\end{array}$ \\
\hline & Ethyl tridecanoate & & $\mathrm{C}_{15} \mathrm{H}_{30} \mathrm{O}_{2}$ & 242 & $\begin{array}{l}\text { Antioxidant (Qurzeddine et } \\
\text { al. 2017) }\end{array}$ \\
\hline
\end{tabular}

electrostatic, van der Waals interaction, and some interacted with positively charged functional groups of an amino acid (lysine, arginine, and histidine), while some interacted with hydroxyl groups of amino acid serine, threonine, and tyrosine. Amino acid residues such as serine, threonine, and tyrosine contribute to rotating hydroxyl groups in the docking complex are considered rigid (Pantsar and Poso 2018).

\subsection{Docking analysis of ligand against SARS-CoV-2 RNA-dependent RNA-polymerase (PDB ID-6M71) target}

A high binding affinity was observed between GC-MS compounds digitoxin, cholesterol margarate, docosanoic acid, pseduosarsasapogenin-5,20-dien, arachidonic amide, $N$ - $\{5$-hydroxy- $N$-pentyl), DI- $N$-decylsulfone, oxiraneundecanoic acid, 3-pentyl, methyl ester, cis, andrographolide, 21-acetoxypregenelone, p-menth-8(10)-en9-ol, 26-hydroxycholesterol, and pregabalin against target protein RNA-dependent RNA-polymerase with high atomic contact energies of $-22.96,-21.60,-13.13$, - 12.54, - 12.04, - 11.57, - 11.38, - 8.43, 8.38, - 7.18, -7.11 , and $-6.85 \mathrm{kcal} / \mathrm{mol}$. The most prominent and common amino acid residues binding to the target proteins were Asn 414, Lys 411, Tyr 141, Val 12, Tyr 546, and Asn 781.

\subsection{Docking analysis of ligands against target SARS-CoV-2 3CL protease (3CL pro) (PDB ID-6M2Q)}

GC-MS compounds of $P$. maculata digitoxin, 26-hydroxycholesterol, 1-methylsulfanyl-9,10-dioxo-dihydro-anthracene-2-carboxylic acid, arachidonic amide, $N$ - $\{5$-hydroxy$N$-pentyl), cholesterol margarate, 21-acetoxypregenelone, pseduosarsasapogenin-5,20-dien, DI- $N$-decylsulfone, Nor-diazepam,3-\{N-hydroxymethyl $\}$ aminocarbonyl, andrographolide, $p$-menth-8(10)-en-9-ol, and pregabalin exhibited high binding affinity $-13,27,-12.61,-12.22$, - 10.86, - 9.91, - 9.22, - 8.41, - 7.39, 7.19, - 7.17, 6.75 , and $-6.33 \mathrm{kcal} / \mathrm{mol}$ against target SARS-CoV-2 3CL protease. The most commonly found amino acid residues binding to the target pocket were Gln 110, Phe 294, and Thr 111 .

\subsection{Docking analysis of ligands against target SARS-CoV-2 post-fusion core structure of spike glycoprotein S2 subunit (PDB ID- 6LXT)}

The target spike glycoprotein-S2 subunit amino acid residues interacted with compounds, 1-methylsulfanyl-9,10-dioxo-9,10-dihydro-anthracene-2-carboxylic, Nor-diazepam, $3-\{N$-hydroxymethyl $\}$ aminocarbonyloxy, andrographolide, 
Table 3 GC-MS analysis of methanol extracts of Pleione maculata

\begin{tabular}{|c|c|c|c|c|c|c|}
\hline $\begin{array}{l}\text { Parts of } P \\
\text { maculata }\end{array}$ & Peak name & Chemical structure & $\begin{array}{l}\text { Molecular } \\
\text { name }\end{array}$ & $\begin{array}{l}\text { Molecular } \\
\text { formula }\end{array}$ & $\begin{array}{l}\text { Molecular } \\
\text { weight }\end{array}$ & Activity \\
\hline \multirow[t]{10}{*}{ leaves } & Phenol, 4-(methoxymethyl)- & & & $\mathrm{C}_{8} \mathrm{H}_{10} \mathrm{O}_{2}$ & 138 & $\begin{array}{l}\text { Anti-diabetic (Balamurugan } \\
\text { et al. 2017) }\end{array}$ \\
\hline & $\begin{array}{l}\text { Pentadecanoic acid, 14- } \\
\text { methyl-, methyl ester }\end{array}$ & & $\begin{array}{l}\text { Palmitic } \\
\text { acid methyl } \\
\text { ester }\end{array}$ & $\mathrm{C}_{19} \mathrm{H}_{34} \mathrm{O}_{2}$ & 270 & $\begin{array}{l}\text { Antioxidant (Vijisaral and } \\
\text { Arumugam 2014) }\end{array}$ \\
\hline & N-hexadecanoic acid & & $\begin{array}{l}\text { Palmitic } \\
\text { acid }\end{array}$ & $\mathrm{C}_{16} \mathrm{H}_{32} \mathrm{O}_{2}$ & 256 & $\begin{array}{l}\text { Anti-inflammatory, } \\
\text { Antioxidant, } \\
\text { hypocholesterolemic } \\
\text { nematicide, pesticide, anti- } \\
\text { androgenic } \\
\text { hemolytic, flavor, } \\
\text { reductase inhibitor (Kumar } \\
\text { et al. 2010; Aparna et al. } \\
2012 \text { ) }\end{array}$ \\
\hline & 9-octadecyne & & $\begin{array}{l}\text { Alkene } \\
\text { compound }\end{array}$ & $\mathrm{C}_{18} \mathrm{H}_{34}$ & 250 & $\begin{array}{l}\text { Antioxidant, Antimicrobial } \\
\text { (Upgade and Bhaskar 2013) }\end{array}$ \\
\hline & Isopropyl linoleate & & & $\mathrm{C}_{21} \mathrm{H}_{38} \mathrm{O}_{2}$ & 322 & $\begin{array}{l}\text { Antioxidant, antidiabetic, } \\
\text { anti-inflammatory } \\
\text { Formulation of skin and hair } \\
\text { care products, facial makeup } \\
\text { (Rajendra et al. 2017; } \\
\text { Rautela et al. 2018) }\end{array}$ \\
\hline & $\begin{array}{l}\text { 6-octadecenoic acid, methyl } \\
\text { ester, (Z)- }\end{array}$ & & $\begin{array}{l}\text { Trans-13- } \\
\text { octadecanoic } \\
\text { acid, methyl } \\
\text { ester } 7\end{array}$ & $\mathrm{C}_{19} \mathrm{H}_{36} \mathrm{O}_{2}$ & 296 & $\begin{array}{l}\text { Anti-inflammatory, } \\
\text { antiandrogenic, cancer } \\
\text { preventive, dermatitigenic } \\
\text { irritant, antiluekotriene-D4, } \\
\text { Hypocholesterolemic, 5- } \\
\text { alpha reductase inhibitor, } \\
\text { anemiagenic, insectifuge, } \\
\text { flavor (Abubakar and } \\
\text { Majinda 2016) }\end{array}$ \\
\hline & $\begin{array}{l}\text { Oxiraneundecanoic acid, 3- } \\
\text { pentyl, methyl ester, cis }\end{array}$ & & & $\mathrm{C}_{19} \mathrm{H}_{36} \mathrm{O}_{3}$ & 312 & $\begin{array}{l}\text { Larvicidal activity } \\
\text { Antioxidant activity } \\
\text { (Elumalai et al. 2015; Al- } \\
\text { Marzoqi 2016) }\end{array}$ \\
\hline & $\begin{array}{l}\text { Octadecanoic acid, methyl } \\
\text { ester }\end{array}$ & & $\begin{array}{l}\text { Methyl } \\
\text { stearate }\end{array}$ & $\mathrm{C}_{19} \mathrm{H}_{38} \mathrm{O}_{2}$ & 298 & $\begin{array}{l}\text { Antifungal and antioxidant } \\
\text { (Pinto et al. 2017) }\end{array}$ \\
\hline & N-hexadecanoic acid & & $\begin{array}{l}\text { Palmitic } \\
\text { acid }\end{array}$ & $\mathrm{C}_{16} \mathrm{H}_{32} \mathrm{O}_{2}$ & 256 & $\begin{array}{l}\text { Anti-inflammatory, } \\
\text { Antioxidant, } \\
\text { hypocholesterolemic } \\
\text { nematicide, pesticide, anti- } \\
\text { androgenic } \\
\text { hemolytic, } \\
\text { reductase inhibitor (Kumar, } \\
\text { et al. 2010; Aparna et al. } \\
2012 \text { ) }\end{array}$ \\
\hline & $\begin{array}{l}\text { Heptacosanoic acid, methyl } \\
\text { ester }\end{array}$ & & $\begin{array}{l}\text { Methyl } \\
\text { heptacosano } \\
\text { ate }\end{array}$ & $\mathrm{C}_{28} \mathrm{H}_{56} \mathrm{O}_{2}$ & 424 & No activity reported \\
\hline \multirow[t]{2}{*}{ Stem } & $\begin{array}{l}\text { Hexadecanoic acid, methyl } \\
\text { ester }\end{array}$ & & & $\mathrm{C}_{17} \mathrm{H}_{34} \mathrm{O}_{2}$ & 270 & $\begin{array}{l}\text { Antibacterial and antifungal } \\
\text { (Abubakar and Majinda } \\
\text { 2016) }\end{array}$ \\
\hline & N-hexadecanoic acid & & $\begin{array}{l}\text { Palmitic } \\
\text { acid }\end{array}$ & $\mathrm{C}_{16} \mathrm{H}_{32} \mathrm{O}_{2}$ & 256 & $\begin{array}{l}\text { Anti-inflammatory, } \\
\text { Antioxidant, } \\
\text { hypocholesterolemic } \\
\text { nematicide, pesticide, anti- } \\
\text { androgenic flavor, } \\
\text { hemolytic, } \\
\text { reductase inhibitor (Kumar } \\
\text { et al. 2010; Aparna et al. } \\
2012 \text { ) }\end{array}$ \\
\hline
\end{tabular}


Table 3 (continued)

\begin{tabular}{|c|c|c|c|c|}
\hline $\begin{array}{l}(2 \mathrm{~s}, 3 \mathrm{~s})-(-)-3-\text { propyloxirane } \\
\text { methanol }\end{array}$ & & $\mathrm{C}_{6} \mathrm{H}_{12} \mathrm{O}_{2}$ & 116 & $\begin{array}{l}\text { Anti-oxidant (Yusufzai et al. } \\
\text { 2019) }\end{array}$ \\
\hline $\begin{array}{l}\text { pyrimidine- } \\
2,4,6(1 \mathrm{H}, 3 \mathrm{H}, 5 \mathrm{H}) \text {-Trione-1- } \\
\text { octadecyl })\end{array}$ & & $\mathrm{C}_{22} \mathrm{H}_{40} \mathrm{~N}_{2} \mathrm{O}_{3}$ & 380 & $\begin{array}{l}\text { compound not reported } \\
2,4,6 \text {-trisubstituted } \\
\text { pyrimidine were evaluated } \\
\text { as anti-malaria drug } \\
\text { (Agrawal et al. 2005) }\end{array}$ \\
\hline 2,3-anhydro-D-galactosan & $\begin{array}{l}\text { Sugar } \\
\text { moeity }\end{array}$ & $\mathrm{C}_{6} \mathrm{H}_{8} \mathrm{O}_{4}$ & 144 & $\begin{array}{l}\text { preservative (Paulpriya et al. } \\
2014 \text { ) }\end{array}$ \\
\hline $\begin{array}{l}\text { 9,12-octadecadienoic acid, } \\
\text { methyl ester }\end{array}$ & & $\mathrm{C}_{19} \mathrm{H}_{34} \mathrm{O}_{2}$ & 294 & $\begin{array}{l}\text { Anticancer, } \\
\text { inflammatory, } \\
\text { antileukotriene, } \\
\text { (Abubakar and } \\
2016)\end{array}$ \\
\hline $\begin{array}{l}\text { 9-octadecenoic acid (Z)-, } \\
\text { methyl ester }\end{array}$ & $\begin{array}{l}\text { Trans-13- } \\
\text { octadecanoic } \\
\text { acid, methyl } \\
\text { ester } 7\end{array}$ & $\mathrm{C}_{19} \mathrm{H}_{36} \mathrm{O}_{2}$ & 296 & $\begin{array}{l}\text { Anti-inflammatory, } \\
\text { antiandrogenic, cancer } \\
\text { preventive, dermatitigenic } \\
\text { irritant, antiluekotriene-D4, } \\
\text { Hypocholesterolemic, 5- } \\
\text { alpha reductase inhibitor, } \\
\text { anemiagenic, insectifuge, } \\
\text { nematicide, pesticide, anti- } \\
\text { androgenic flavor, } \\
\text { hemolytic, 5-alpha } \\
\text { reductase inhibitor (Kumar } \\
\text { et al. 2010; Aparna et al. } \\
\text { 2012) }\end{array}$ \\
\hline $\begin{array}{l}(2 \mathrm{~s}, 3 \mathrm{~s})-(-)-3-\text { propyloxirane } \\
\text { methanol }\end{array}$ & & $\mathrm{C}_{6} \mathrm{H}_{12} \mathrm{O}_{2}$ & 116 & $\begin{array}{l}\text { Anti-oxidant (Yusufzai et al. } \\
\text { 2019) }\end{array}$ \\
\hline $\begin{array}{l}\text { pyrimidine- } \\
2,4,6(1 \mathrm{H}, 3 \mathrm{H}, 5 \mathrm{H}) \text {-Trione-1- } \\
\text { octadecyl })\end{array}$ & & $\mathrm{C}_{22} \mathrm{H}_{40} \mathrm{~N}_{2} \mathrm{O}_{3}$ & 380 & $\begin{array}{l}\text { compound not reported } \\
2,4,6 \text {-trisubstituted } \\
\text { pyrimidine were evaluated } \\
\text { as anti-malaria drug } \\
\text { (Agrawal et al. 2005) }\end{array}$ \\
\hline 2,3-anhydro-D-galactosan & $\begin{array}{l}\text { Sugar } \\
\text { moeity }\end{array}$ & $\mathrm{C}_{6} \mathrm{H}_{8} \mathrm{O}_{4}$ & 144 & $\begin{array}{l}\text { preservative (Paulpriya et al. } \\
2014 \text { ) }\end{array}$ \\
\hline $\begin{array}{l}\text { 9,12-octadecadienoic acid, } \\
\text { methyl ester }\end{array}$ & & $\mathrm{C}_{19} \mathrm{H}_{34} \mathrm{O}_{2}$ & 294 & $\begin{array}{l}\text { Anticancer, } \\
\text { inflammatory, } \\
\text { antileukotriene, } \\
\text { (Abubakar and } \\
2016)\end{array}$ \\
\hline
\end{tabular}


Table 3 (continued)

\begin{tabular}{|c|c|c|c|c|c|}
\hline & $\begin{array}{l}\text { 9-octadecenoic acid (Z)-, } \\
\text { methyl ester }\end{array}$ & $\begin{array}{l}\text { Trans-13- } \\
\text { octadecanoic } \\
\text { acid, methyl } \\
\text { ester } 7\end{array}$ & $\mathrm{C}_{19} \mathrm{H}_{36} \mathrm{O}_{2}$ & 296 & $\begin{array}{l}\text { Anti-inflammatory, } \\
\text { antiandrogenic, cancer } \\
\text { preventive, dermatitigenic } \\
\text { irritant, antiluekotriene-D4, } \\
\text { Hypocholesterolemic, 5- } \\
\text { alpha reductase inhibitor, } \\
\text { anemiagenic, insectifuge, } \\
\text { flavor (Abubakar and } \\
\text { Majinda 2016) }\end{array}$ \\
\hline & $\begin{array}{l}\text { Octadecanoic acid, methyl } \\
\text { ester }\end{array}$ & $\begin{array}{l}\text { Methyl } \\
\text { stearate }\end{array}$ & $\mathrm{C}_{19} \mathrm{H}_{38} \mathrm{O}_{2}$ & 298 & $\begin{array}{l}\text { Antifungal and antioxidant } \\
\text { (Pinto et al. 2017) }\end{array}$ \\
\hline & $\begin{array}{l}\text { Triacontanoic acid, methyl } \\
\text { ester }\end{array}$ & $\begin{array}{l}\text { Methyl } \\
\text { triacontanate }\end{array}$ & $\mathrm{C}_{31} \mathrm{H}_{62} \mathrm{O}_{2}$ & 466 & $\begin{array}{l}\text { Antimicrobial (Kumar et al. } \\
\text { 2015) }\end{array}$ \\
\hline Roots & $\begin{array}{l}\text { Hexadecanoic acid, methyl } \\
\text { ester }\end{array}$ & & $\mathrm{C}_{17} \mathrm{H}_{34} \mathrm{O}_{2}$ & 270 & $\begin{array}{l}\text { Antibacterial and antifungal } \\
\text { activity (Abubakar and } \\
\text { Majinda 2016) }\end{array}$ \\
\hline & 9,12 -octadecadienal & & $\mathrm{C}_{18} \mathrm{H}_{32} \mathrm{O}$ & 264 & $\begin{array}{l}\text { Antimicrobial and } \\
\text { antioxidant (Gurnani et al } \\
2015 \text { ) }\end{array}$ \\
\hline & E,E-1,9,17-Docasatriene & $\begin{array}{l}\text { alkene } \\
\text { compound }\end{array}$ & $\mathrm{C}_{22} \mathrm{H}_{40}$ & 304 & 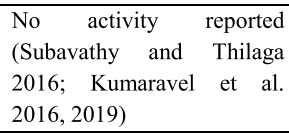 \\
\hline & $\begin{array}{l}\text { 9-octadecenoic acid, methyl } \\
\text { ester }\end{array}$ & $\begin{array}{l}\text { Trans-13- } \\
\text { octadecanoic } \\
\text { acid, methyl } \\
\text { ester } 7\end{array}$ & $\mathrm{C}_{19} \mathrm{H}_{36} \mathrm{O}_{2}$ & 296 & $\begin{array}{l}\text { Anti-inflammatory, } \\
\text { antiandrogenic, cancer } \\
\text { preventive, dermatitigenic } \\
\text { irritant, antiluekotriene-D4, } \\
\text { Hypocholesterolemic, 5- } \\
\text { alpha reductase inhibitor, } \\
\text { anemiagenic, insectifuge, } \\
\text { flavor (Abubakar and } \\
\text { Majinda 2016) }\end{array}$ \\
\hline & Nonanal & aldehyde & $\mathrm{C}_{9} \mathrm{H}_{18} \mathrm{O}$ & 142 & $\begin{array}{l}\text { Anti-aging use in cosmetics } \\
\text { Fungicides (Plainfosse et al. } \\
\text { 2017; Zhang et al. 2017) }\end{array}$ \\
\hline & pregabalin & & $\mathrm{C}_{8} \mathrm{H}_{17} \mathrm{NO}_{2}$ & 159 & $\begin{array}{l}\text { Adjunctive treatment of } \\
\text { partial seizures, use in } \\
\text { neuropathic pain, neuralgia, } \\
\text { use in alcohol withdrawal } \\
\text { syndrome, restless leg } \\
\text { syndromes, migraine and } \\
\text { vasomotor symptoms of } \\
\text { menopause (World Health } \\
\text { Organization 2018) }\end{array}$ \\
\hline & $\begin{array}{l}\text { Octadecanoic acid, methyl } \\
\text { ester }\end{array}$ & $\begin{array}{l}\text { Methyl } \\
\text { stearate }\end{array}$ & $\mathrm{C}_{19} \mathrm{H}_{38} \mathrm{O}_{2}$ & 298 & $\begin{array}{l}\text { Antifungal and antioxidant } \\
\text { (Pinto et al. 2017) }\end{array}$ \\
\hline & $\begin{array}{l}\text { Tetracosanoic acid, methyl } \\
\text { ester }\end{array}$ & $\begin{array}{l}\text { Methyl } \\
\text { lignocerate }\end{array}$ & $\mathrm{C}_{25} \mathrm{H}_{50} \mathrm{O}_{2}$ & 382 & $\begin{array}{l}\text { Antibacterial, } \\
\text { Antimicrobial (Valiei et al. } \\
\text { 2011; Ukil et al. 2015) }\end{array}$ \\
\hline & $\begin{array}{l}\text { Nor-Diazepam,3-\{(N- } \\
\text { hydroxymethyl)aminocarbo } \\
\text { nyl oxy\} }\end{array}$ & & $\begin{array}{l}\mathrm{C}_{18} \mathrm{H}_{16} \mathrm{CIN}_{3} \\
\mathrm{O}_{4}\end{array}$ & 373 & No activity reported \\
\hline
\end{tabular}


digitoxin, cholesterol margarate, 26-hydroxycholesterol, p-menth-8(10)-en-9-ol, and pregabalin with high binding affinity (ACE) of $-10.95,-9.23,-8.93,-8.23,-8.23$, 6.68 , and $6.29 \mathrm{kcal} / \mathrm{mol}$. The most common residues binding to target proteins were Ser 943, Ser 940, Glu 1188, Ser 939, Asp 1184, Asp 936, Lys 1191, Gln 935, and Asn 1187.

\subsection{Drug-likeness analysis of bioactive compound}

Drug-likeness was analyzed to check whether bioactive compounds possess favorable ADME (absorption, distribution, metabolism, and excretion) properties. Drug-likeness compounds should have a good aqueous solubility which is predicted by three methods ESOL, (ALI) $\operatorname{logS}$, and (SILICOS-IT) $\log$ S (Shweta and Rashmi 2019). Orally active drug should obey Lipinski five rule in Table 5, molecular weight (MW) not more than $500 \mathrm{~g} / \mathrm{mol}$, hydrogen bond acceptors not more than 10 , hydrogen bond donors not more than $5, \log P$ value less than 5 , and number of rotatable bonds not less than 10 (Lipinski et al. 1997), and a violation of two or more rule depicts a molecule as not orally active. Drug-likeness analysis of bioactive compounds listed in Table 6 with their different parameters is shown in SwissADME bioavailability radar in Fig. 7. Nor-diazepam, $3-\{N$ hydroxymethyl $\}$ aminocarbonyloxy bioactive compound showed good binding affinity against SARS-CoV-2 with high drug-likeness parameters such as good solubility and no excretion problems as there is no pharmacokinetics P-gp (permeability glycoprotein) interference and non-inhibitor of CYP enzymes and compound is specific in nature (zero alerts for PAINS (pan assay interference compounds)). 1-Methylsulfanyl-9,10-dioxo-9,10-dihydro-anthracene2-carboxylic acid drug-likeness parameters is also good, since it follows Lipinski RO5, Ghose, Veber, Egan, Muegge rule, and Bioavailability score 0.55 but the PAINS (Pan assay interference compounds) exhibited some interference with Quinone A compound which indicates compound is not specific and is moderately soluble in nature. Digitoxin might have shown good binding affinity but does not obey any of the drug-likeness parameters. Pregabalin and $p$-menth-8(10)-en-9-ol bioactive compounds are highly soluble in nature but depict one or two violations as druglikeness due to lower molecular weight $(<160)$. 21-Acetoxypregenolone an antisense target compound showing good binding affinity against SARS-CoV-2 shows moderate drug-likeness parameters, specific in nature, and no excretion problems but is an inhibitor of CYP2C9 enzyme. Pseduosarsasapogenin-5, 20-dien, and andrographolide drug-likeness activity are moderate with good solubility property. Compounds such as DI- $N$-decylsulfone, arachidonic amide, $N$ - $\{5$-hydroxy- $N$-pentyl $\}, 26$-hydroxycholesterol, cholesterol margarate, and docosanoic acid showed poor solubility with various violations as a drug molecule.
Arabidiol an already marketed antiviral drug does follow Lipinski RO5 but does not obey drug-likeness parameters and is poorly soluble compared to bioactive compounds of Pleione maculata with high solubility in nature.

\section{Discussion}

The GC-MS analysis of other orchids mostly focuses on flower scent profile rather than a therapeutic profile, for example, Vanda species (Darmasiwi et al. 2015), Rhynchostylis gigantean Ridl, Rhynchostylis gigantean var. harrisonianum Holtt., Vanda coerulea and Dendrobium parishii Rchb. F., (Julsrigival et al. 2013) and Dendrophylax lindenii (Sadler et al. 2011). In this paper, the GC-MS report of Pleione maculata mainly focused on total medicinal important compounds present in different parts. Compounds such as phenol 4-(ethoxymethyl), heptacosanoic acid methyl ester, 9-octadecanoic acid (Z)- 2-hydroxy-1-(hydroxymethyl) ethyl ester (Vijisaral and Arumugan 2014; Rajalakhsmi and Mohan 2016), nonanoic acid 9(3-hexemyldenecycyclopropylidene2-hydroxy-1-1 (HYD) (Sahin et al. 2006), alpha-ketostearic acid ethyl ester, 1-naphthaleneproponal alpha-ethyl decahydro-5-(hydroxymethyl), cis, cis, cis-7,10,13-hexadecatrienal (Prabhadevi et al. 2012; Abdulaziz et al. 2019), 1H-purin2-amine-6-methoxy, 4-cyanobenzoic acid Tridec-2-YNYL ester, alpha- $L$-fucopyranose 1,2.3,4-bis (benzeneboonate), cholesterol margarate, 2.pyridinecarboxylic acid 6-methoxy, 1-methyl sulfanyl -9-10-dioxo-9,10-dihydro-anthracene-2-carboxylic acid, pyrimidine-2,4,6(1H,3H,5H)-trione-1-octadecyl), E,E-1,9,17-docasatriene (Subavathy and Thilaga 2016; Kumaravel et al. 2019), $N$-decyl-alpha $D$-2-deoxyglycoside, cholesterol isocaproate, 7.dehydrocholesterol isocaproate, and pyrimidine-2,4,6(1H,3H,5H)-trione-1-octadecyl) are a few of the compounds which were identified but have no bioactivity reported so far. Compounds such as 8-oxatetracyclo $\{5 \cdot 2 \cdot 1 \cdot 1(2,6)$. 1(4,10) $\}$ dodecane, 7-tertbutyl-1,9,9-trimeth, docosane, 2,4-dimethyl, kryptogenin 2,4-dinitrophenyl hydrazine, $N$-decyl-alpha, $D$-2-deoxyglycoside are some novel compounds which have not been reported earlier. Phytochemical studies and antimicrobial activity of Pleione maculata were also reported which depicts the presence of various phytochemical compounds in different parts of epiphyte and antibacterial activity showed a distinct zone of inhibition against Streptococcus pneumonia (Sympli et al. 2019).

Docking involves the interaction of various polar and non-polar groups, and both play a significant role in the stability of protein-ligand interactions. In docking, water solvent is removed by most programs as they tend to form hydrogen bonds with molecules either as donor or acceptor (Pantsar and Poso 2018). Protein-ligand interactions are strong on the removal of $\mathrm{H}$-bond but in association 


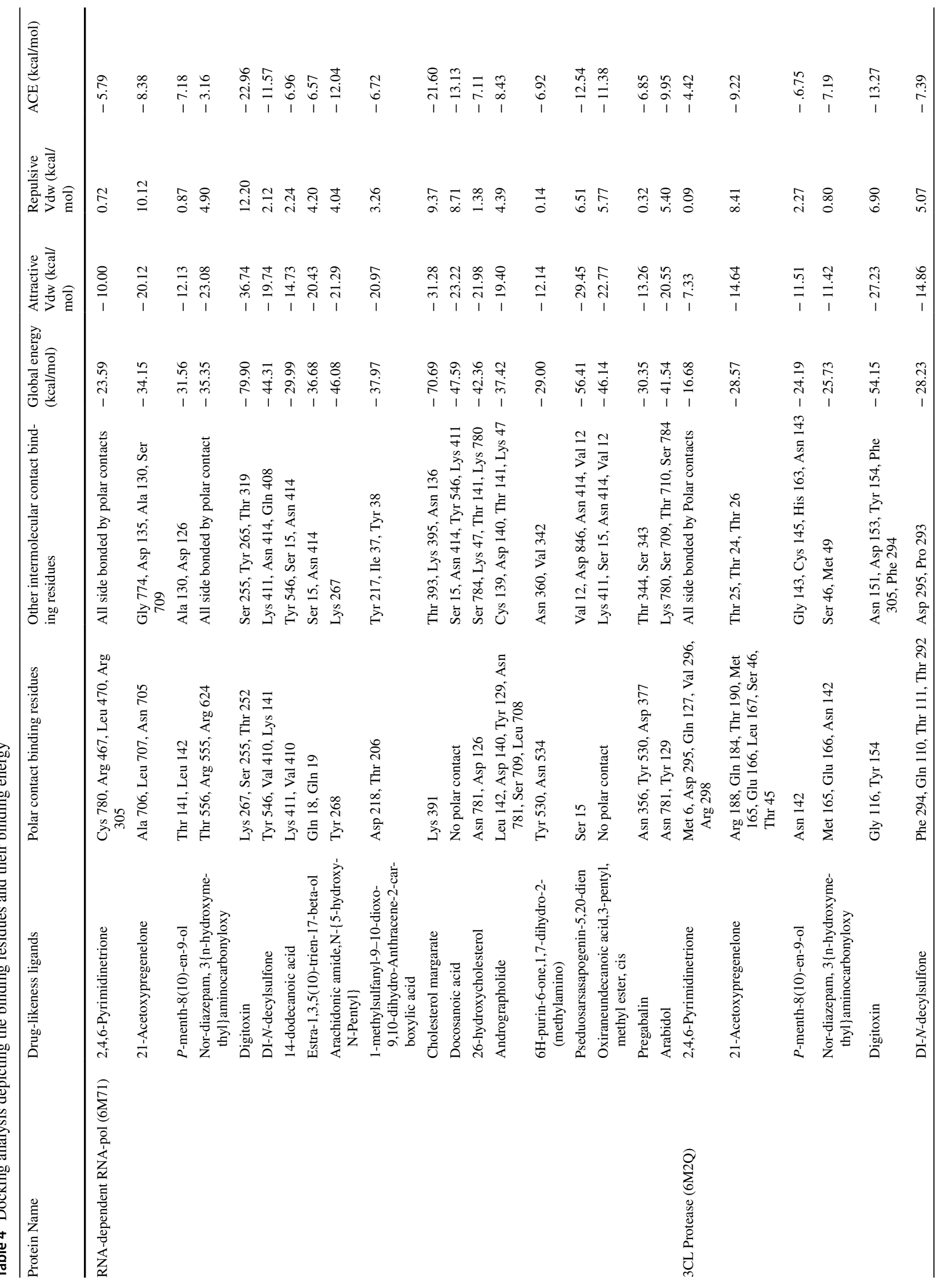




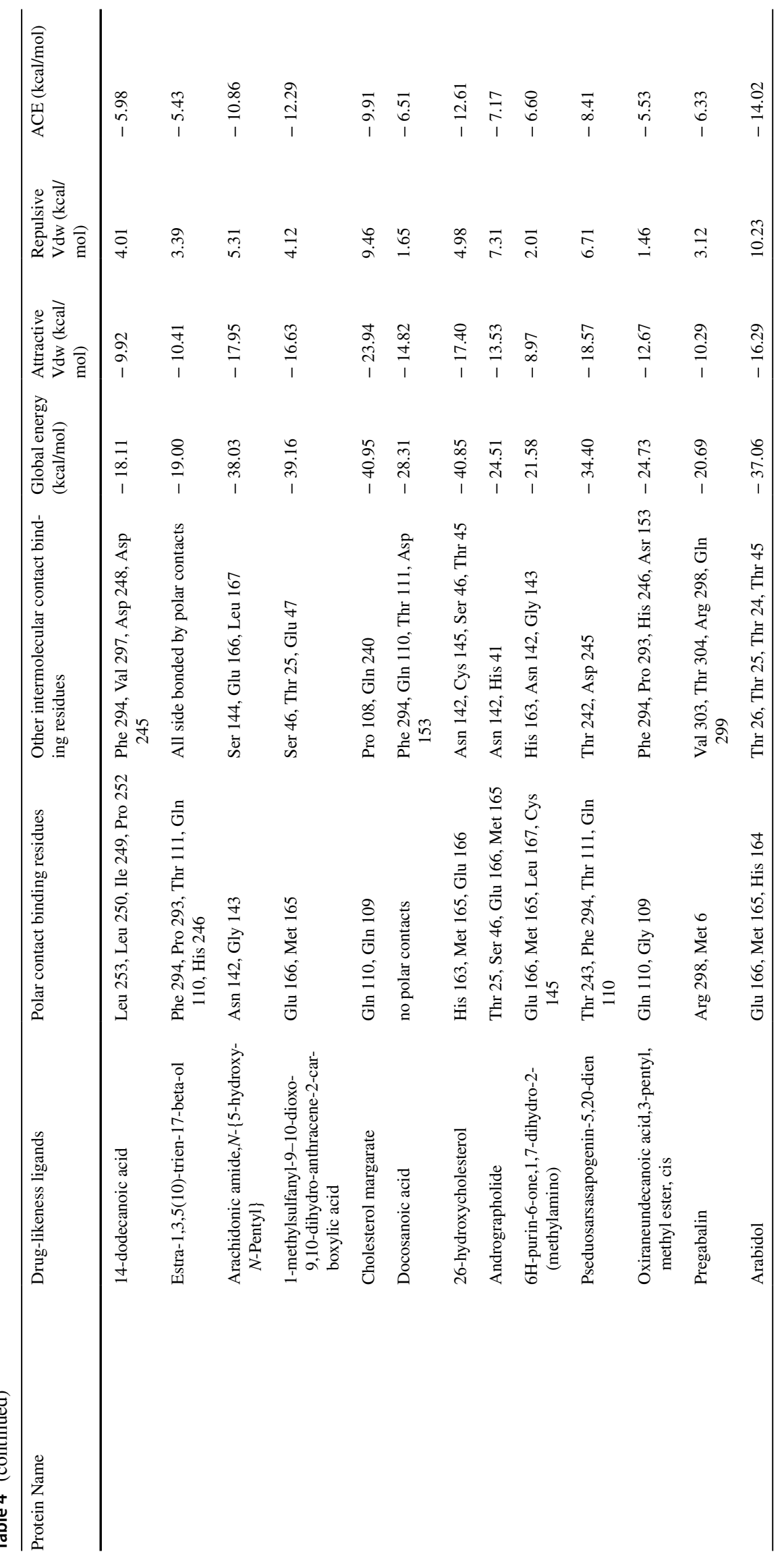




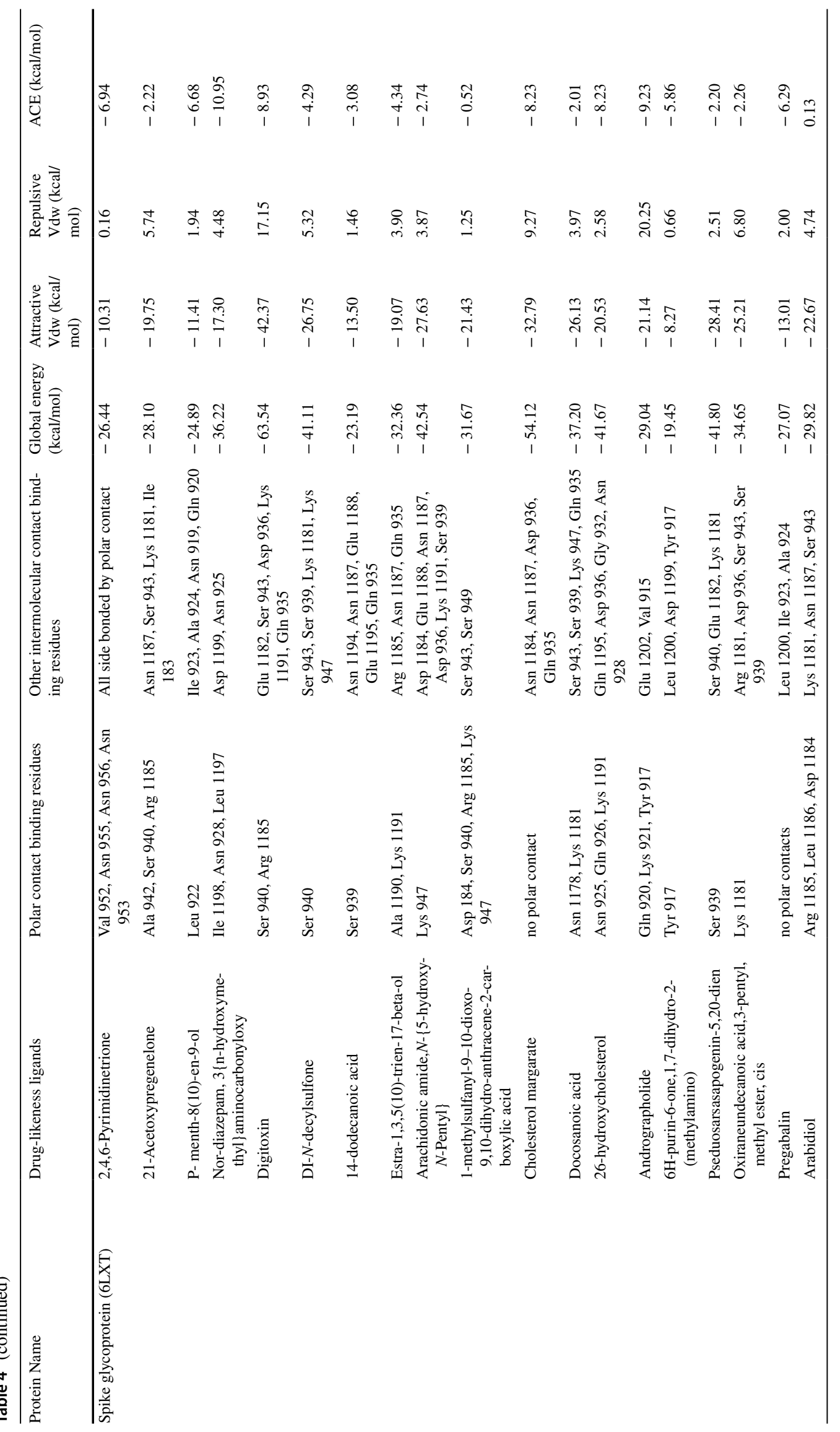



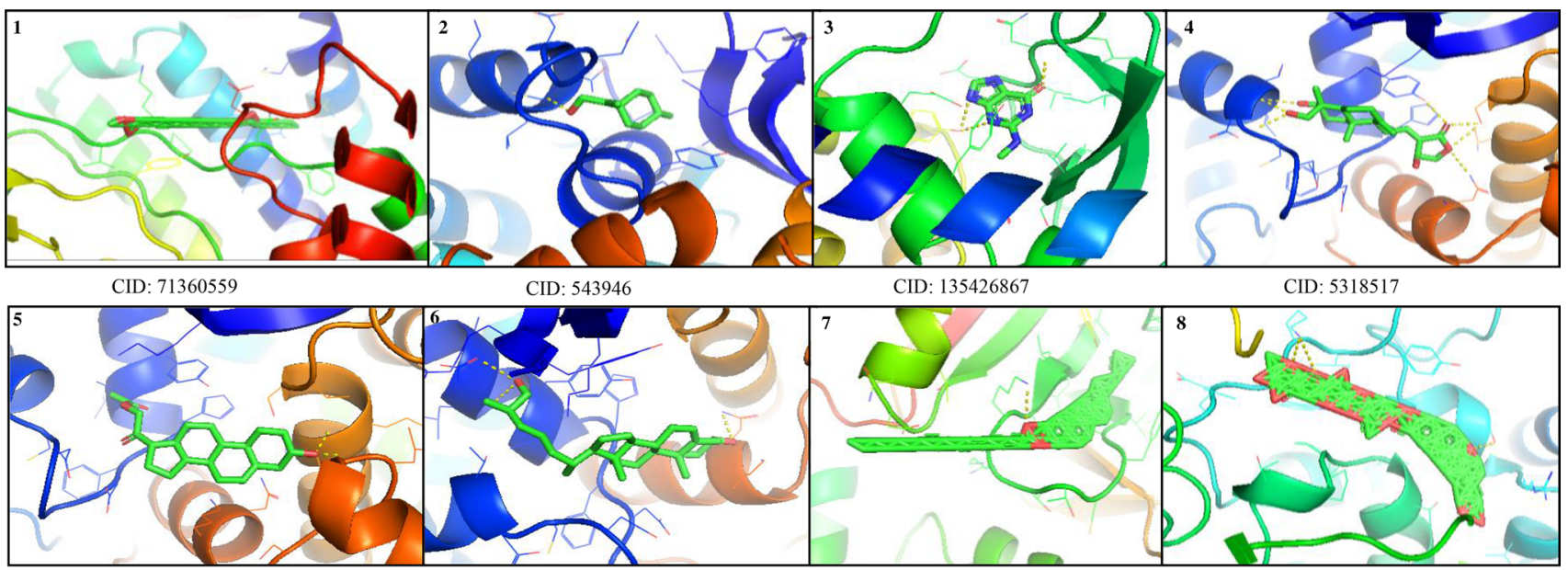

CID: 248856

CID: 99470

CID: 14077841

CID: 441207

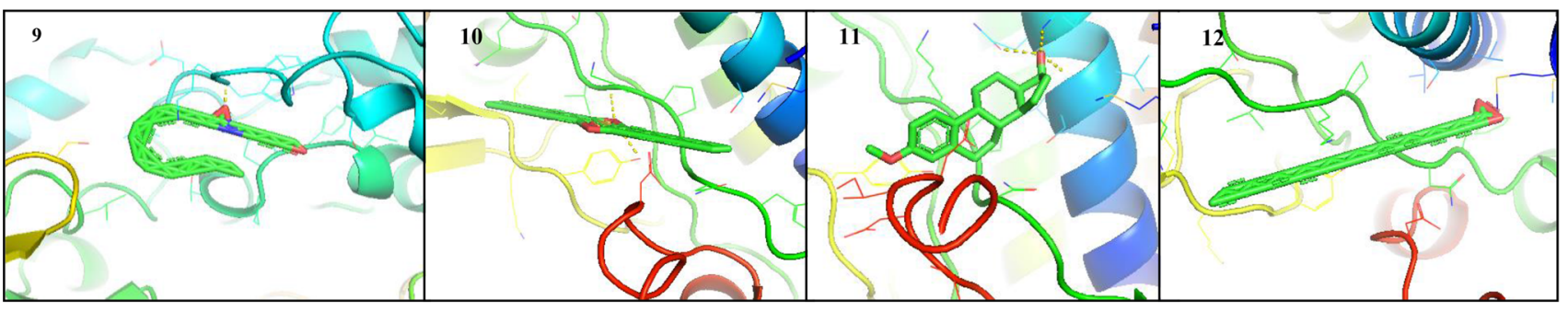

CID: $5283405 \quad$ CID: 19089489

CID: 13948

CID: 8215

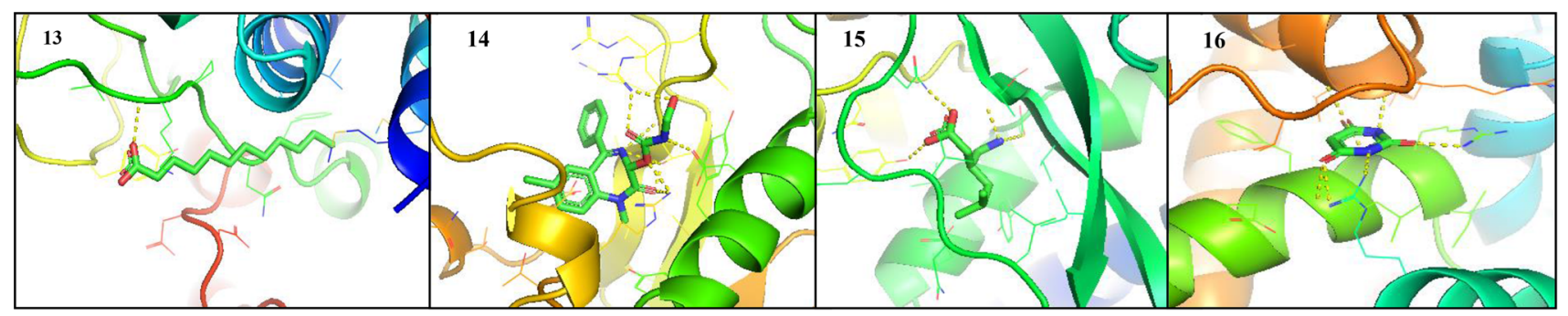

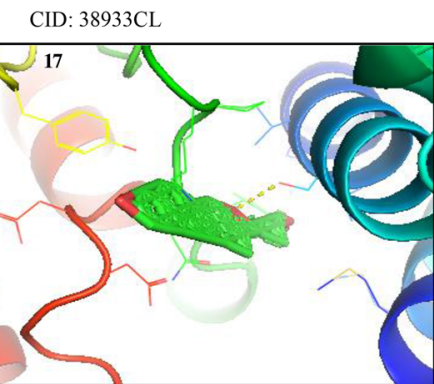

CID: 261799

CID: 541761

CID: 5486971

CID: 6211

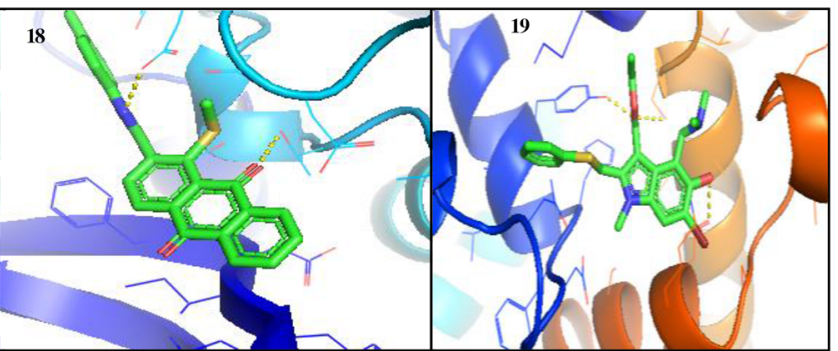

CID: 572031

Fig. 4 Molecular docking of target SARS-CoV-2 RNA-dependent RNA-polymerase (PDB ID- 6M71) with the S-CoV-2 RNA-dependent RNA polymerase (PDB ID-6M71) with the GC-MS bioactive compounds of Pleione maculata (1) oxiraneundecanoic acid,3-pentyl, methyl ester, cis (2) p-menth-8(10)-en-9-ol (3) 6H-purin-6-one,1,7dihydro-(2-methylamino) (4) andrographolide (5) 21-acetoxypregenelone (6) 26-hydroxycholesterol (7) cholesterol margarate (8) digitoxin (9) arachidonic amide, $N$-\{5-hydroxy- $N$-pentyl $\}$ (10) DI$N$-decylsulfone (11) estra-1,3,5(10)-trien-beta-ol (12) docosanoic acid (13) 14-dodecanoic acid (14) Nor-diazepam,3-\{N-hydroxymethyl $\}$ aminocarbonyloxy (15) pregabalin (16) 2,4,6-pyrimidinetrione (17) pseduosarsasapogenin acid, 3-pentyl, methyl ester, cis, (18) 1-methylsulfanyl-9,10-dioxo-9, 10-dihydro-anthracene-2-carboxylic acid and positive control (19) arabidiol with other non-covalent interactions (electromagnetic interactions, ionic interactions, van der Waals interaction, and hydrophobic interactions) play an important role in protein-ligand stability (Yadav et al. 2017). van der Waals interactions are non-covalent repulsive or attractive intermolecular interactions, bonding energy decreases from zero to 


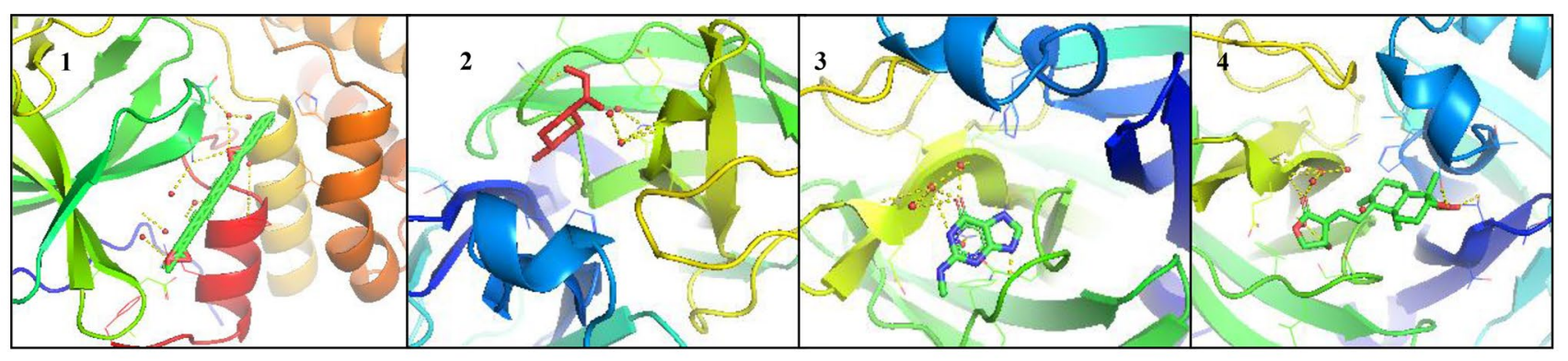

$$
\text { CID: } 71360559
$$

CID: 543946

CID: 135426867

CID: 5318517
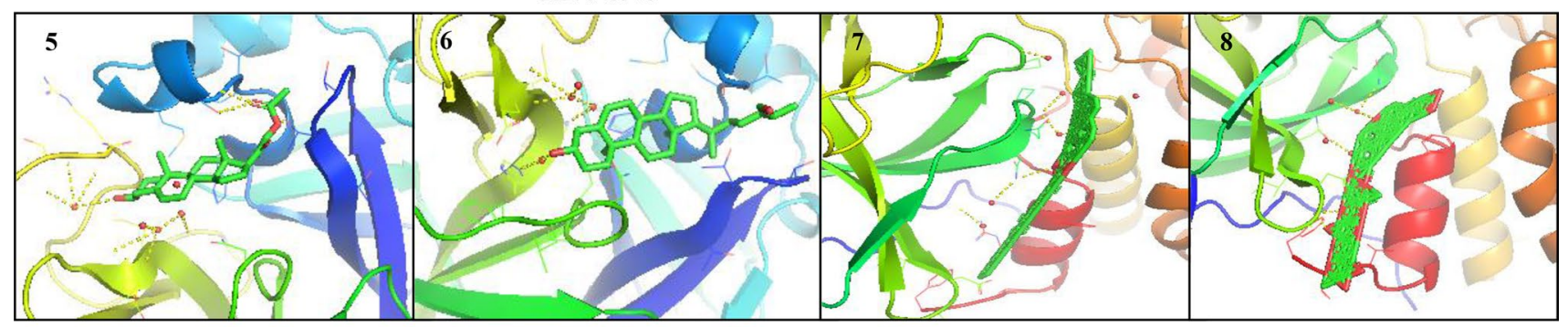

CID: 248856

CID: 99470

CID: 14077841

CID: 441207
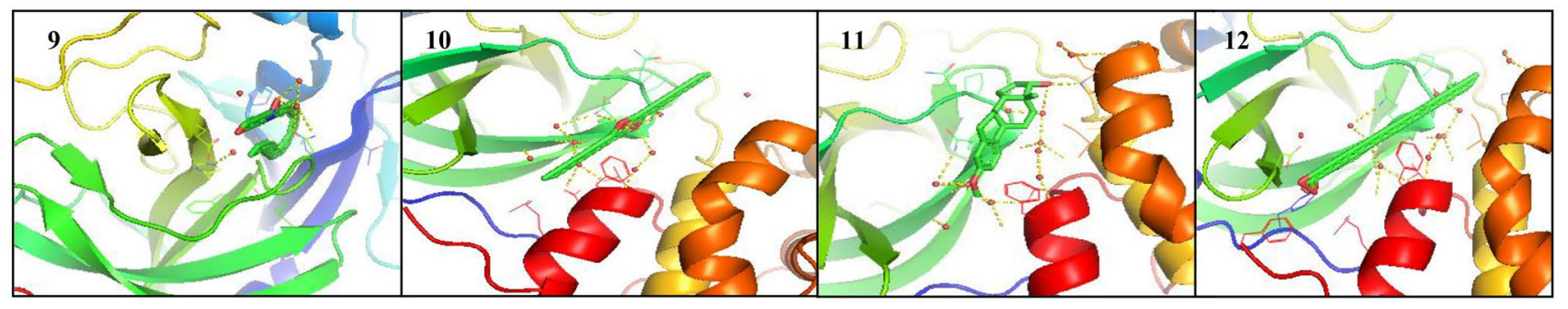

CID 5283405

CID 19089489

CII) 13948

CII 8215
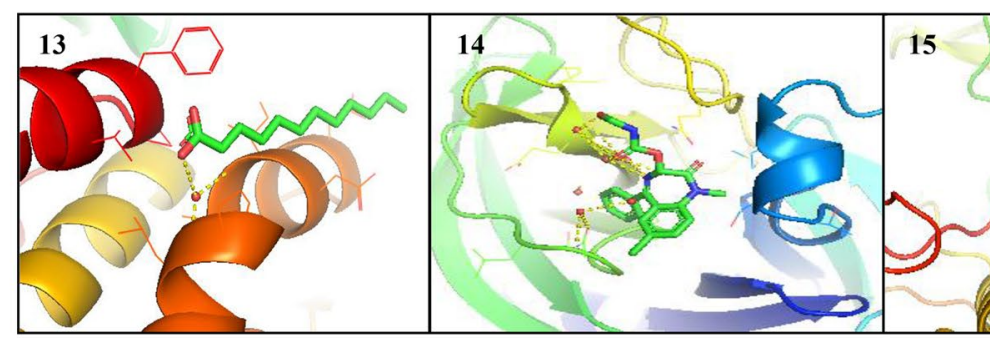

CID: 38933CL

CID: 541761

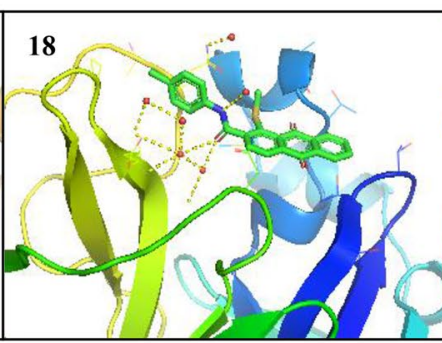

CID: 572031

Fig. 5 Molecular docking of target SARS-CoV-2 3CL protease (PDB ID- 6M2Q) with the GC-MS bioactive compounds of Pleione maculata (1) oxiraneundecanoic acid,3-pentyl, methyl ester, cis (2) p-menth-8(10)-en-9-ol (3) 6H-purin-6-one,1,7-dihydro(2-methylamino) (4) andrographolide (5) 21-acetoxypregenelone (6) 26-hydroxycholesterol (7) cholesterol margarate (8) digitoxin (9) arachidonic amide, $N$-\{5-hydroxy- $N$-pentyl $\}$ (10) DI- $N$-decyl- sulfone (11) estra-1,3,5(10)-trien-beta-ol (12) docosanoic acid (13) 14-dodecanoic acid (14) Nor-diazepam,3-\{N-hydroxymethyl $\}$ aminocarbonyloxy (15) pregabalin (16) 2,4,6-pyrimidinetrione (17) pseduosarsasapogenin acid, 3-pentyl, methyl ester, cis, (18) 1-methylsulfanyl-9,10-dioxo-9, 10-dihydro-anthracene-2-carboxylic acid and positive control (19) arabidiol 

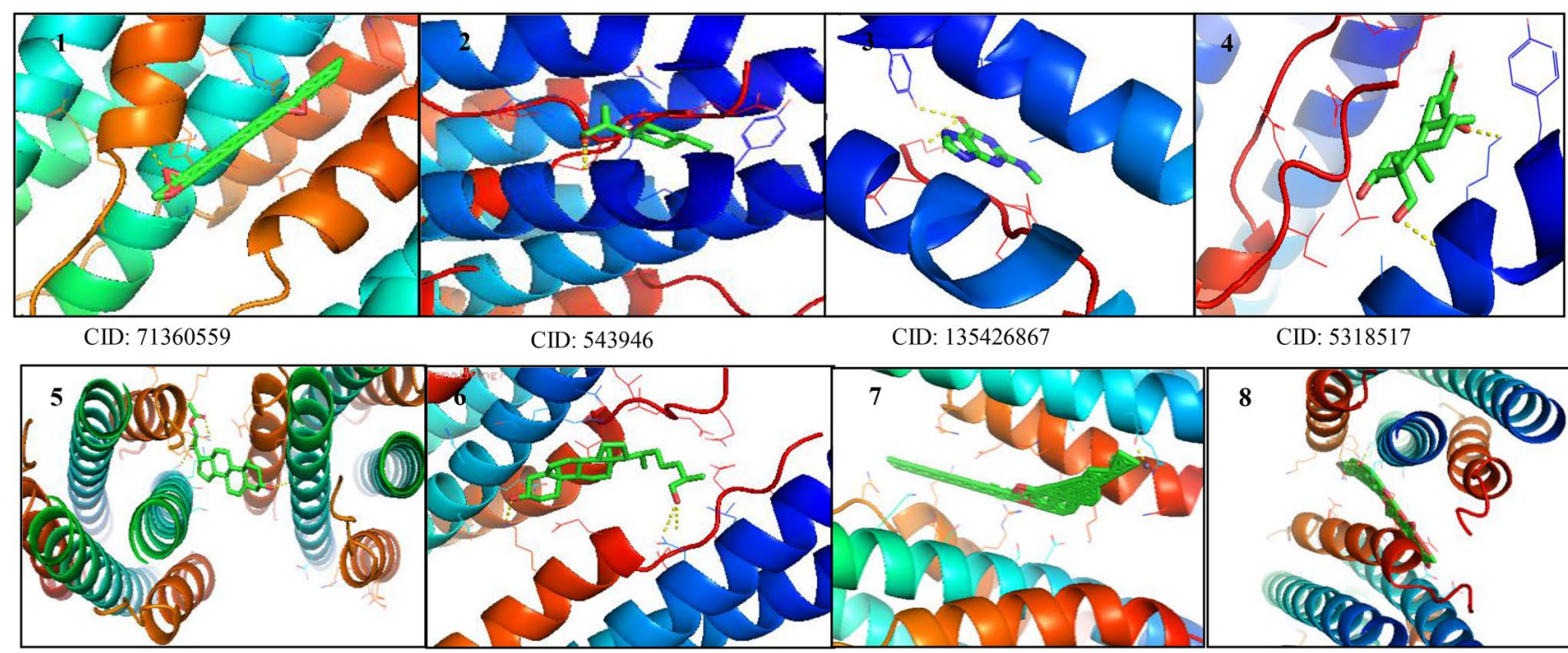

CID: 543946

CID: 135426867

CID: 5318517

CID: 248856
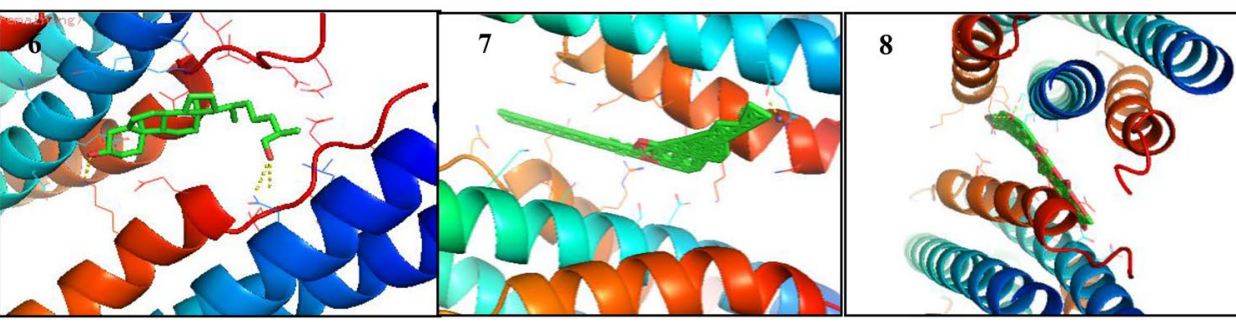

CID: 99470

CID: 14077841

CID: 441207

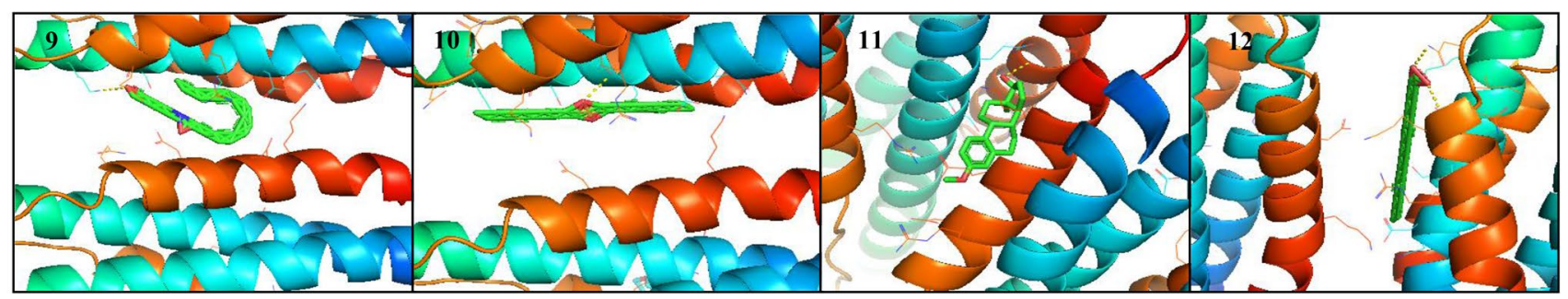

CID: 5283405

CID: 19089489

CID: 13948

CID: 8215

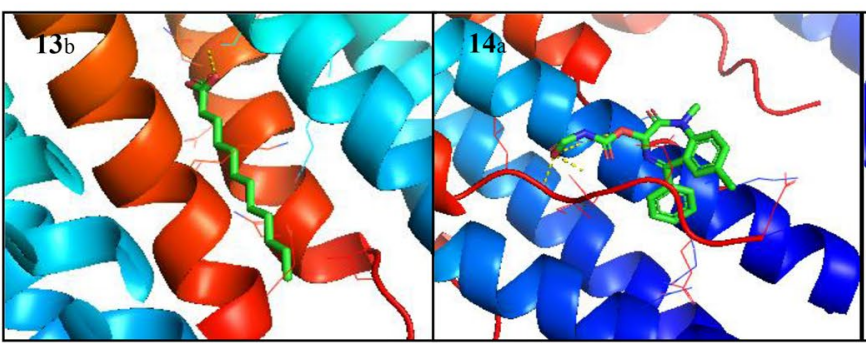

CID: 38933CL

CID: 541761

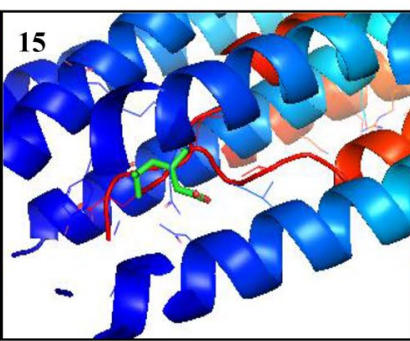

CID: 5486971

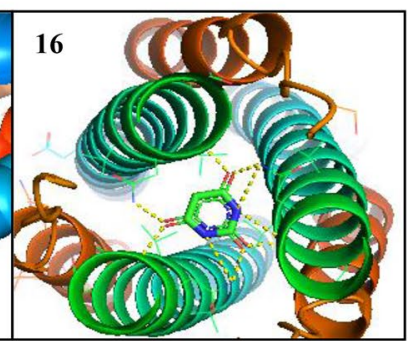

CID: 6211

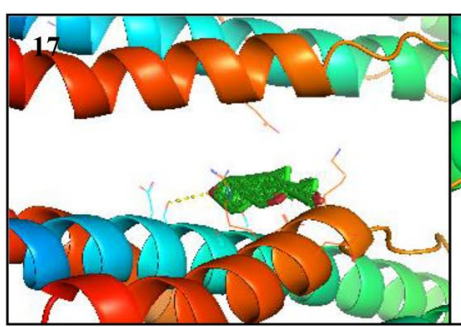

CID: 261799

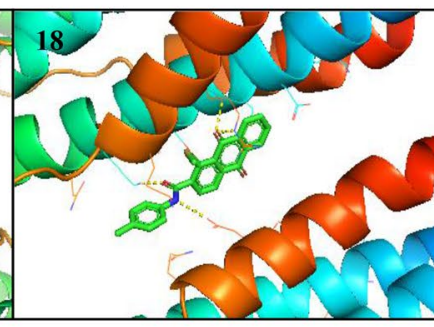

CID: 572031

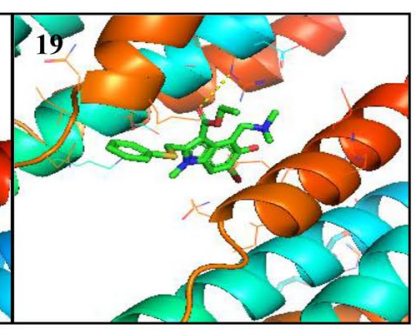

CID: 131411

Fig. 6 Molecular docking of target SARS-CoV-2 spike glycoprotein S2 subunit (PDB ID- 6LXT) with the GC-MS bioactive compounds of Pleione maculata (1) oxiraneundecanoic acid,3-pentyl, methyl ester, cis (2) p-menth-8(10)-en-9-ol (3) 6H-purin-6-one,1,7-dihydro(2-methylamino) (4) andrographolide (5) 21-acetoxypregenelone (6) 26-hydroxycholesterol (7) cholesterol margarate (8) digitoxin (9) arachidonic amide, $\mathrm{N}$-\{5-hydroxy- $\mathrm{N}$-pentyl $\}$ (10) DI- $N$-decyl- sulfone (11) estra-1,3,5(10)-trien-beta-ol (12) docosanoic acid (13) 14-dodecanoic acid (14) Nor-diazepam,3-\{N-hydroxymethyl $\}$ aminocarbonyloxy (15) pregabalin (16) 2,4,6-pyrimidinetrione (17) pseduosarsasapogenin acid, 3-pentyl, methyl ester cis, (18) 1-methylsulfanyl-9,10-dioxo-9, 10-dihydro-anthracene-2-carboxylic acid and positive control (19) arabidiol 
Table 5 Lipinski rule of five (RO5) violation

\begin{tabular}{ll}
\hline Molecular weight & $\begin{array}{l}<500 \text { Dal- } \\
\text { tons }\end{array}$ \\
\hline Hydrogen bond donors & No $>5$ \\
Hydrogen bond acceptors & No $>10$ \\
Octanol-water partition coefficient $(\log \mathrm{P})$ & $<5$ \\
Number of rotatable bonds & No $<10$ \\
\hline
\end{tabular}

its negative value when the distance of attraction between two molecules is close, and repulsive forces occurs when the distance of separation decreases and bonding energy increases (Singh 2016). van der Waals interaction may be considered weak, but they play a vital role in structure and biomolecules interaction. Hydrogen bonds are also weak non-covalent bond which induces thermal fluctuation in energies and tend to form or break rapidly causing conformational changes during binding (Bronowska 2011). GC-MS bioactive compound Nor-diazepam, $3-\{N$-hydroxymethyl $\}$ aminocarbonyloxy, andrographolide, depicts a high binding affinity of -10.95 and $-9.23 \mathrm{Kcal} / \mathrm{mol}$ and very good druglikeness properties when compared to the binding affinity of fisetin, quercetin, isorhamnetin, genistein, luteolin, resveratrol, and apigenin with $-8.5,-8.5,-8.3,-8.2,-8.2$, $-7.9,-7.7 \mathrm{kcal} / \mathrm{mol}$, respectively, against $\mathrm{S} 2$ subunit spike glycoprotein (Rane et al. 2020). The atomic contact energy (ACE) was also reported higher in tea flavonoid catechin products epigallocatechin and theaflavin gallate without re-docking refinement used (Maiti and Banerjee 2020). A docking complex of epitope-based peptide vaccine component and toll-like receptor-5 (TLR-5) of SARS-COV-2 spike glycoprotein exhibits good binding affinity which might have the potential to activate immune cascades for destroying viral antigens (Bhattacharya et al. 2020). Molecular docking between structure-based drug design PC786 bearing ChEMBL ID 4291143 against SARS-COV-2 S protein and $\mathrm{M}^{\text {pro }}$ revealed a good binding affinity ranging from -1.5 to $11.5 \mathrm{kcal} / \mathrm{mol}$ (Panda et al. 2020). Pleione maculata compounds Nor-diazepam,3-\{N-hydroxymethyl $\}$ aminocarbonyloxy, andrographolide, pregabalin, $P$-menth-8(10)-en-9-ol, 21-acetoxypregenolone, and 1-methylsulfanyl-9,10-dioxo9,10-dihydro-anthracene-2-carboxylic acid are few druglikeness compounds obeying the Lipinski RO5 properties such as solubility, lipophilicity $(\log \mathrm{P})$ less than 5, hydrogen bond not more than 5 , good solubility, and absorption. The drug-likeness parameters described are the main criteria in primary drug development. The bioactive compounds of $P$. maculata screened using GC-MS have more potential as drug-likeness against various intractable diseases, for example, here SARS-CoV-2 pneumonia (COVID-19) because of its stronger binding affinity, closer interaction, and abide by ADME characteristics.

\section{Conclusions}

GC-MS analysis in methanol, ethanol, and acetone extracts of different parts (leaves, stem, and roots) of P. maculata identified major peaks indicating the presence of phytochemical constituents such as palmitic acid, vitamin E, vitamin A precursor, cardiac glycoside, ascorbic acid, linoleic acid, oleic acid, stearic acid, alkaloid, di-terpenoid, flavonoid, and phenolic and steroidal compound. Cholestenone compound which has the capability to suppress fat accumulation and antiobesity activity is not commonly found in other medicinal plants but reported in GC-MS study of $P$. maculata. The identified compounds were known to have various activities commonly antimicrobial, anti-inflammatory, antioxidant, cancer preventive, hypocholesterolemic, nematicide, antiasthma, antiarrhythmic activity (improve heartbeat), antiviral activity, larvicidal activity, anticonvulsants, and antiobesity activities. Molecular docking analysis is a structure-based design of drugs. The compounds of $P$. maculata showed a highly effective binding affinity (atomic contact energy). The spike glycoprotein $\mathrm{S} 2$ subunit of SARS-CoV-2 was one rigid target as it initiates membrane fusion with host receptor but bioactive compound Nor-diazepam, $3-\{N$-hydroxymethyl $\}$ aminocarbonyloxy exhibits good docking and high binding affinity with atomic contact energy $-10.95 \mathrm{kcal} / \mathrm{mol}$ and very high drug-likeness properties. For centuries, plants are the main source of medicinally important natural products. The paper represents the first report on GC-MS analysis, molecular docking, and drug-likeness study on P. maculata. The findings are expected to contribute a significant and major therapeutic impact in the pharmaceutical and nutraceutical companies. An in vitro and in vivo analysis has to be implemented to understand the mechanism of action, cytotoxicity studies on the above effective bioactive compounds. In conclusion, a study on rare Pleione maculata highlights their prospective therapeutic potentialities against various intractable diseases and their bioactive components will enhance a sustainable rural livelihood in both primary and secondary health care and also to save them from extinction and over-exploitation. The effective drug-likeness compound does not have to be separated or isolated directly from the source plant, but the study will provide a basic idea on a synthetic production of effective bioactive compounds. 


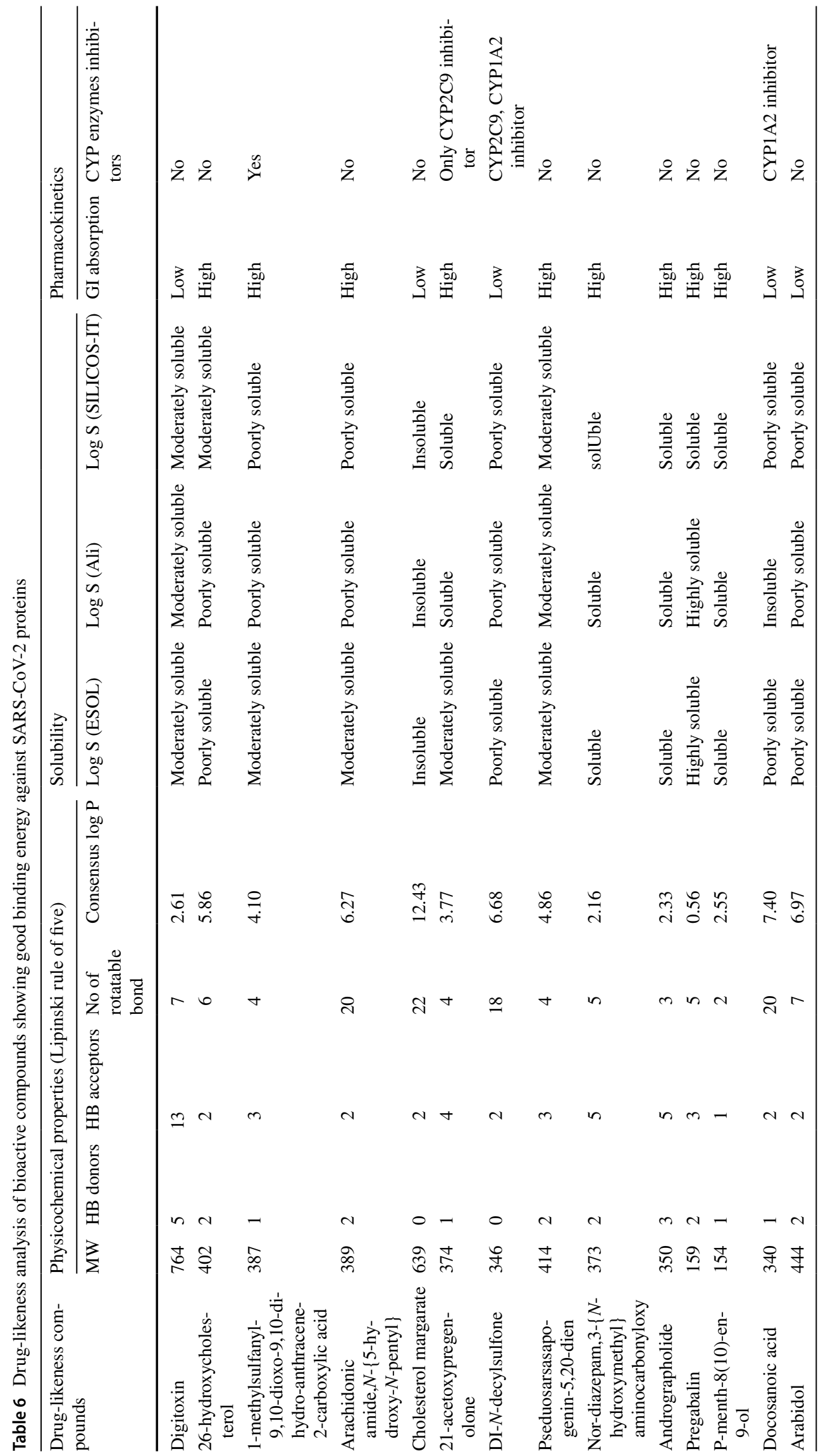




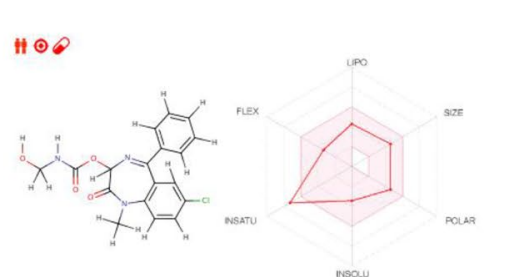

Nor-diazepam,3-\{N-

hydroxymethyl aminocarbonyloxy
100

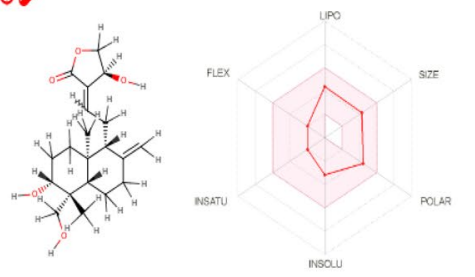

Andrographolide
$11 \odot \odot$

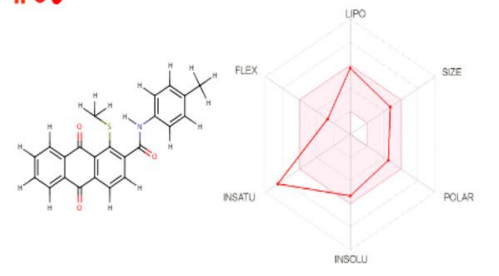

1-methylsulfanyl-9,10-dioxo-9,10-dihydroanthracene-2-carboxylic acid

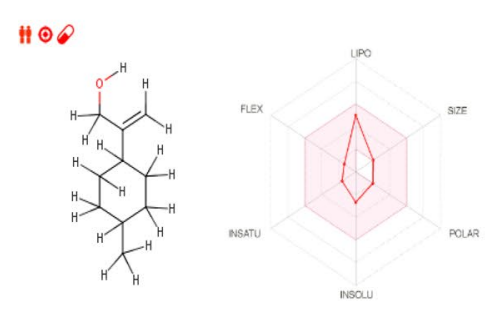

P-menth-8(10)-en-9-ol

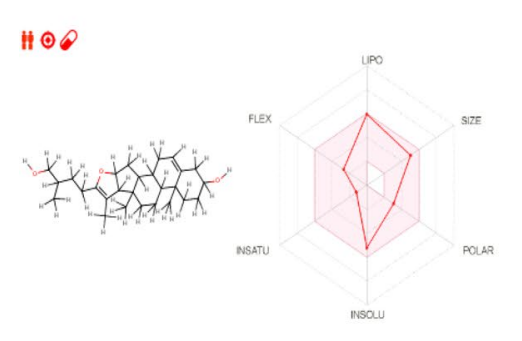

Pseduosarsasapogenin-5,20-dien

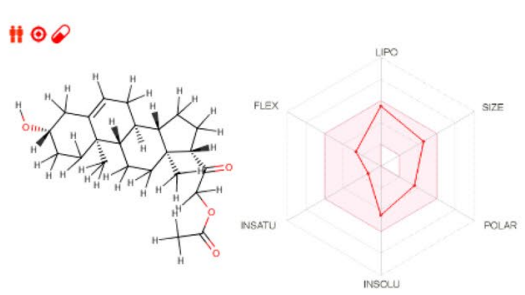

21-acetoxypregenolone

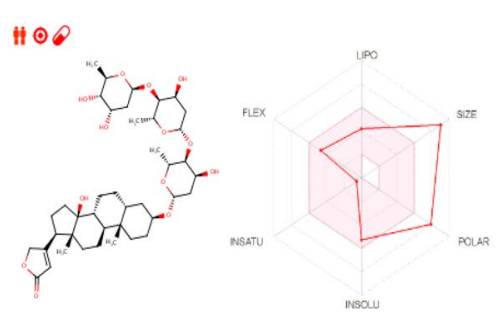

Digitoxin

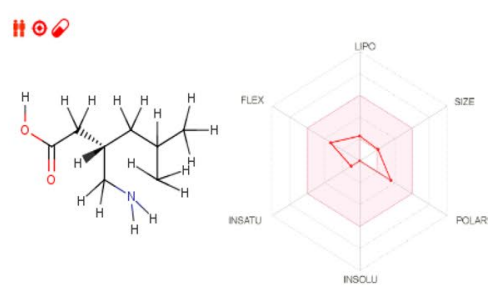

Pregabalin

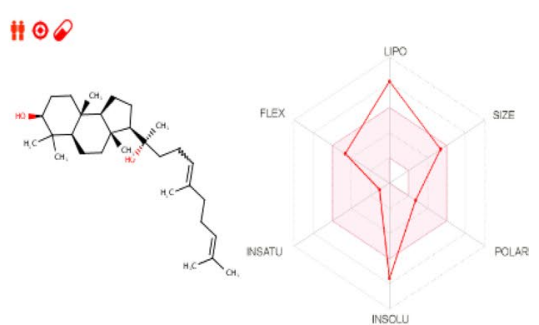

Arabidiol

Fig. 7 SwissADME bioavailability radar of different bioactive drug-likeness molecules, where the pink areas represent each property (lipophilicity, molecular weight, solubility, and flexibility)

Supplementary Information The online version contains supplementary material available at https://doi.org/10.1007/s13721-020-00276-1.

Acknowledgements I thank Dr. Mahua Gupta Choudhury, MSc. dissertation guide, and Dr. Vedant V. Borah, doctoral research guide, for their support. I would also like to thank Dr. Supriyo Sen for introducing me to Indian Institute of Technology (IIT), Biotech Park Guwahati, Assam, for GC-MS analysis. I sincerely acknowledge the School of Biosciences, Assam Don Bosco University, for permitting and providing me with basic requirements for the methodology part. Lastly, I acknowledge Dr. Rajiv Chandra Dev Goswami, IIT, Guwahati, for the guidance and information on GC-MS analysis. The work is financially supported by Ministry of tribal affair, Govt. of India (Ref: 201819-NFST-MEG-00850).

\section{Compliance with ethical standards}

Conflict of interest The author declares no conflict of interest. The research work is original.

\section{References}

Abdulaziz AA, Dabar MLG, Manting MM, Torres MAJ, Aranas AT, Mindo RAR, Cabrido CK, Demayo CG (2019) Qualitative evaluation of the antimicrobial, antioxidant and medicinally important phytochemical constituents of the ethanolic extracts of the leaves of Gliricidia sepium (JACQ) WALP. Pharmacophore 10(4):72-83

Abubakar NM, Majinda TRR (2016) GC-MS analysis amd Preliminary antimicrobial activity of Albizia adianthifolia (Schumach) and Pterocarpus angolensis (DC). Medicines 3(3):1-9. https://doi. org/10.3390/medicines3010003

Adeoye-Isijola OM, Olajuyigbe OO, Jonathan GS, Coopoosamy MR (2018) Bioactive compounds in ethanol extract of Lentinus squarrosulus Mont- A Nigerian medicinal macrofungus. Afr J Complement Altern Med 15(2):42-50. https://doi.org/10.21010 /ajtcamv $15 \mathrm{i} 2.6$

Agboke AA, Attama AA (2016) Bioactive components and antibacterial activities of n-Hexane extract of Moringa oleifera root 
bark on clinical isolates of methicillin resistant Staphylococcus aureus. Int J Curr Res Chem Pharm Sci 3(3):1-9

Agrawal A, Srivastava K, Puri SKK, Chauhan PMS (2005) Synthesis of 2,4,6-trisubstituted pyrimidines as anti-malarial agents. Bio Med chem 13(5):4645-4650. https://doi.org/10.1016/j. bmc.2005.04.061

Al-Gara' awi NI, (2019) Analysis of bioactive phytochemical compound of (Cyperus alternifolius L.) By using gas chromatography- mass spectrometry. IOP Conf Ser Mater Sci Eng 571:1-19. https://doi. org/10.1088/1757-899X/571/1/012047

Al-Marzoqi AH, Hadi MY, Hameed IH (2016) Determination of metabolites products by Cassia angustifolia and evaluate antimicrobial activity. J Pharm Phytotherapy 8(2):25-48. https://doi. org/10.5897/JPP2015.0367

Alagammal M, Soris PT, Mohan VR (2012) GC-MS analysis of Polygala rosmarinifolia Wights \& Arn. J App Pharm Sci 2(4):188190. https://doi.org/10.7324/JAPS.2012.2504

Anand K, Ziehbuhr J, Wandhwani P, Meters JR, Hilgenfield R (2003) Coronavirus main proteinase (3CL pro) structure: basis for design of anti-SARS. Sciencexpress 300(5626):1763-1767. https ://doi.org/10.1126/cience.1085658

Andrusier N, Nussinov R, Wolfson HJ (2007) FireDock: Fast iteration refinement in molecular Docking. Proteins 69(1):139-159. https ://doi.org/10.1002/prot.21495

Ansarali S, Manikandan S, Lakshmanan GMA (2018) Identification of biological components from potential bone healer medicinal plants. J Drug Del Ther 8(3):32-41. https://doi.org/10.22270/ jddt.v8i3.1762

Aparna V, Dileep VK, Mandal KP, Karthe P, Sadasivan C, Haridas M (2012) Anti-inflammatory property of n-Hexadecanoic acid: structural evidence and kinetic assessment. Chem Biol Drug Design 80(3):434-439. https://doi.org/10.111 1/j.1747-0285.2012.01418.x

Asaraja A, Sahayaraj K (2013) Screening of insecticidal activity of brown macroalgal extracts against Dysdercus cingulatus (Fab.) (Hemiptera: Pyrrhocoridae). J Biopest 6(2):193-203

Autore G, Marzocco S, Palladino C, Saturnino C, Sinicropi S, Spagnuolo A, Vivacqua E, Capasso A (2010) New amides of arachidonic acid as potential anti-inflammatory drugs: a preliminary study. Biomed Res 21(4)

Babar AG, Pande A, Kulkarni BG (2016) Anti-fungal activity and investigation of bioactive compounds of marine intertidal bivalve Gafrarium divaricatum from West coast of India. Int J Pure App Bio 4(2):211-217. https://doi.org/10.18782/2320-7051.2273

Balamurugan TSB, Gunanithi M, Raja I, Vinothkumar D, Ramesh Babu NG, Sumanthi S, Aneesh N, Florida T (2017) Antidiabetic potential of various traditional medicinal plants and in silico validation. Eur J Biotech Biosci 5(2):34-40

Balasubramani S, Sabapathi G, Moola AK, Solomon RV, Venuvanalingam P, Diana RKB (2018) Evaluation of the leaf essential oil from Artemisia vulgaricus and its larvicidal and repellent activity against dengue fever vector Aedesaegypti- an experimental and molecular docking investigation. Am Chem Soc Pub 3:1565715665. https://doi.org/10.1021/acsomega.8b01597

Barry OP, Practico D, Lawson JA, FitzGerald GA (1997) Trancellular activation of platelets and endothelial cells by bioactive lipids in platelet microparticles. J Clin Invest 99(9):2118-1227

Bhattacharya M, Sharma AR, Patra P, Ghosh P, Sharma G, Patra BC, Lee S, Chakraborty C (2020) Development of epitopebased peptide vaccine against novel coronavirus 2019 (SARSCOV-2): Immunoinformatics approach. Med Virol 92:618631. https://doi.org/10.1002/jmv.25736

Bronowska AK (2011) Thermodynamics of ligand-protein interaction. Implication for molecular design thermodynamicinteraction studies-solid, liquid and gases. In Dr. Juan Carlos
Moreno pirajajn (ed) InTech. Thermodynamics. 1-48. https:// doi.org/10.5772/19447

Chandrasekar T, Rao KRM, Kumar VR, Prabhu K, Kumar NS, Divya D (2015) GC-MS analysis, antimicrobial, antioxidant activity of an Ayurvedic medicine, Nimbapatradi Choornam. J Chem Pharm Res 7(8):124-136

Chauhan S, Sharma S (2017) Conservation of rare and endangered therapeutically important orchid Pleione maculata (Lindl.) Lindl. \& Paxton through pseudobulb culture In-vitro. Ind J Sci Res 15(1):22-26

Chen C, Tong Z, Liao D, Li Y, Yang G, Li M (2014) Chemical composition and antimicrobial and DPPH scavenging activity of essential oil of Toona sinensis (A. Juss) Roem from China. BioResources 9(3):5262-5278

Chidambaram N, Zhou L, Cohen JS (1996) Targeting of antisense: synthesis of steroid-linked and steroid-bridged oligonucleotides. Drug Deliv 3(1):27-33. https://doi.org/10.3109/10717 549609031378

Chidambarampillai ST, Mohan VR (2013) GC-MS analysis of bioactive components of aerial parts of Fluggea leucopyrus Willd (Euphorbiaceae). J App Pharm Sci 3(5):126-130

Da Silva DLL, Nascimento DSM, Silva SHD, Furlan M, Bolzani SV (2003) Antibacterial activity of a stearic acid derivative from Stemodia foliosa. Planta Med 68(12):1137-1139

Daina A, Michielin O, Zoete V (2003) SwissADME: a free web tool to evaluate pharmacokinetics, drug-likeness and medicinal chemistry friendliness of small molecules. Nat Sci Rep. https ://doi.org/10.1038/srep42717

Darmasiwi S, Indriani V, Innata D, Semiarti E (2015) The potential production of Aromatic Compounds in Flowers of Vanda tricolor. In: The 5th International conference on Mathematics and Natural Sciences 090006- 1- 090006-4. https://doi. org/10.1063/1.4930751. Accessed 25 Apr 2020

Das NU, Madhavi N (2011) Effect of polyunsaturated fatty acids on drug-sensitive and resistant tumor cells in vitro. Lipids Health Dis 10(159):1-35. https://doi.org/10.1186/1476-511X-10-159

De Kock N (2016) Targeted analysis of bioactive steroids and oxycholesterols. Method Dev Appl 1:41

De LC, Medhi RP (2015) Orchid-A diversified component of farming systems for profitability and livelihood security of small and marginal farmers. J Glob Biosci 4(2):1393-1406

De Sousa DP, Raphael E, Brocksom U (2007) Sedative effect of monoterpene alcohols in mice: a preliminary screening. Zeit fur Nat C J Biosci. https://doi.org/10.1515/znc-2007-7-816

Dinesh MD, Carmel A, George N, Ajma N, Anjana JC, Meenatchisundaram S (2016) Terminaliachebula-A potential natural herbal drug against mastitis isolates. IOSR J Agri Vet Sci 9(10):64-69

Doss CGP, Chakraborty C, Chen L, Zhu H (2014) Integrating In silico prediction methods, molecular docking and molecular dynamics simulation to predict the impact of ALK Missense mutations in structural perspective. BioMed Res Int 2014:115. https://doi.org/10.1155/2014/895831

Dreosti IE (1996) Bioactive ingredients: antioxidants and polyphenols in tea. Nutrit Rev 54(11):S51-S58. https://doi. org/10.1111/j.1753-4887.1996.tb03819.x

Dua S, Garg N (2013) Biochemical methods of analysis. Narosa Publishing House, New Delhi

Duhovny D, Nussinov R, Wolfson HJ (2002) Efficient unbound docking of rigid molecules. Algorith Bioinform Springer Ver 2452:185-200. https://doi.org/10.1007/3-540-45784-4_14

Easwaran L, Ramani AV (2014) Phytochemical examination and GC-MS studies of the medicinal plant- Naravelia zeylanica. Int J Res Dev Pharm Life Sci 3(5):1180-1188 
Ekor M (2014) The growing use of herbal medicines: issues relating to adverse reactions and challenges in monitoring safety. Front Pharm 4(177):1-10. https://doi.org/10.3389/fphar.2013.00177

Elaiyaraja A, Chandramohan G (2016) Comparative phytochemical profile of Indoneesiella echioides (L.) Nees leaves using GCMS. J Pharm Phytochem 5(6):158-171

Elumalai D, Hemalatha P, Kaleena PK (2015) Larvicidal activity and GC-MS analysis of Leucas aspera against Aedes aegyptiAnopheles stephensi and Culex quinquefasciatus. J Saud Soc Agri Sci 16(4):306-313. https://doi.org/10.1016/j.jssas.2015.10.003

Eswaraiah G, Peele KA, Krupanidhi S, Kumar BR, Venkateswarulu TC (2020) Identification of bioactive compounds in leaf extract of Avicennia alba by GC-MS analysis and evaluation of its invitro anticancer potential against MCF7 and HeLa cell lines. J King Saud Univ-Sci 32(1):740-744. https://doi.org/10.1016/j. jksus.2018.12.010

Ezekiel A, Zubai S, Abiodun O, Iqbal J (2018) GC-MS analysis and anti-diabetic potentials of Bridelia micrantha crude extracts. Integr Mol Med 5(4):1-5

Fang Li (2016) Strcuture, function, and evolution of Coronavirus spike protein. Annu ReV Virol 3(1):237-261. https://doi.org/10.1146/ annurev-virology-110615-042301

Gastner T, Krimmer PH (2013) US Patent 8,501,810 B2; 2013. Date of patent- 6 Aug 2013; $1-8$

George LO, Radha HR, Somasekariah BV (2018) In vitro anti-diabetic activity and GC-MS analysis of bioactive compounds present in the methanol extract of Kalanchoe pinnata. Ind $\mathrm{J}$ Chem 57B:1213-1221

Ghosal S, Srivastava KA (2013) Fundamentals of Bioanalytical techniques and instrumentation. Raj Press, New Delhi

Gideon AV (2015) GC-MS analysis of phytochemical components of Pseudoglochidion anamalayanum Gamble: an endangered medicinal tree. As J Plant Sci Res 5(12):36-41

Gopinath S, Sakthidevi G, Muthukumaraswamy S, Mohan VR (2013) GC-MS Analysis of bioactive constitutes of Hypericum mysorense (Hypericaceae). J Curr Chem Pharm Sci 3(1):6-15

Groneberg DA, Hilgenfield R, Zabel P (2005) Molecular mechanism of severe acute respiratory syndrome (SARS). Resp Res 6(8):1-16. https://doi.org/10.1186/1465-9921-6-8

Gulam M, Ali NK, Kamal MK, Mayur P (2016) Antisense therapy and its application in biopharmaceutical research: past, present and future. As Pac J Pharm App Sci 61-69

Guo F, Cheng Li S, Wang L, Zhu D (2012) Protein-protein binding site identification by enumerating the configurations. BMC Bioinform 13(158):1-25. http://www.biomedcentral. com/1471-2105/13/158

Gurnani N, Gupta M, Mehta D, Mehta KB (2015) Chemical composition, total phenolic and flavonoid contents, and in vitro antimicrobial and antioxidant activities of crude extracts from red chilli seeds (Capsicum frutescens L.). J Taib Univ Sci. https:// doi.org/10.1016/j.jtusci.2015.06.011

Hadi MY, Mohammed GJ, Hameed IH (2015) Analysis of bioactive chemical compounds of Nigella sativa using Gas chromatography-mass spectrometry. J Pharm Phytotherapy 8(2):8-24. https ://doi.org/10.5897/JPP2015.0364

Hall DC, Ji H-F (2020) A search for medications to treat COVID-19 via in silico molecular docking models of the SARS-CoV-2 spike glycoprotein and 3CL protease. Trav Med Inf Dis. https://doi. org/10.1016/j.tmaid.2020.101646

Hamada HM, Awad M, El-Hefny M, Moustafa MAM (2018) Insecticidal activity of garlic (Allium sativum) and ginger (Zingiber officinale) oils on the cotton leaf worm, Spodoptera littoralis (Boisd) (Lepidoptera:Noctuidae). Afri Entomo 26(1):84-94. https://doi.org/10.4001/003.026.0084

Hameed IH, Altameme HJ, Idan SA (2016) Artemisia annua: bioachemical products analysis of methanolic aerial parts extract and anti-microbial capacity. Res J Pharm Biol Chem Sci 7(2):1843-1868

Hussein MH (2016) Analysis of trace heavy metals and volatile chemical compounds of Lepidium sativum using atomic absorption spectroscopy, gas chromatography-mass spectrometric and fourier-transform infared spectroscopy. Res J Pharm Biol Chem Sci 7(4):2529-2555

Ingole NS (2016) Phytochemical analysis of leaf extract of Ocimum americanum L. (Lamiaceae) by GC-MS method. World scientific news 76-87

Iyamah P, Famuti A, Idu M (2017) GC-MS and molecular docking studies for identification of anti-malarial compounds in PMII-a polyherbal formulation. Chem Res J 2(1):46-56

Jacobson M (1969) Sex pheromone of the pink bollworm moth: biological masking by its geometrical isomer. Science 163(3863):190 191. https://doi.org/10.1126/science.163.3863.190

Jahan Y, Mahmood T, Bagga P, Kumar A, Singh K, Mujahid M (2015) Future prospects of cough treatment, herbal medicines v/s modern drugs. Int J Pharm Sci Res 6(9):1000-1009

Javitt NB (1990) 26-Hydroxycholesterol: synthesis, metabolism and biologic acitivities. J Lipd Res 31:1527-1533

Jialal I, Grundy MS (1992) Effect of dietary supplementation with alpha-tocopherol on the oxidative modification of low density lipoprotein. J Lipd Res 33(6):899-906

Jothi SR, Uttayakumari F, Bharathy V (2015) Phytochemical profile of leaf samples of subspecies of Senna italic Mill. Bioscience Discovery 6(2):106-111

Julsrigival J, Songsak T, Kirdmanee C, Chansakaow S (2013) Determination of volatile constituents of Thai fragrant orchids by gas chromatography-mass spectrometry with solid-phase microextraction. J Nat Sci 12(1):43-56

Karthi S, Vinothkumar M, Karthic U, Manigandan V, Saravanan R, Vasantha-Srinivasan P, Kamaraj C, Shivakumar MS, De Mandal S, Velusamy A, Krutmuang P, Senthil-Nathan S (2020) Biological effects of Avicennia marina (Forssk.) Vierh Extractson physiological, biochemical, and anti-microbial activitiesagainst three challenging mosquito vectors and microbial pathogens. Env Sci Pol Res. https://doi.org/10.1007/s11356-020-08055-1

Karunanithi A, Venkatachalam S (2019) Optimization of ultrasoundassisted extraction of phenolic compounds from wood apple pulp: identification of phytochemical using GC-MS. Chem Ind Chem Eng Quart 25(4):361-368

Khalil MN, Shalaby AE, Ali AIMD, Ali ME, Aboul-Enein MA (2014) Biological activities of secondary metabolites from Emericella nidulans EGCU 312. Afri J Microbiol Res 8(20):2011-2021. https://doi.org/10.5897/AJMR2014.6827

Khan K, Firdous S, Ahmad A, Muhammad N, Rasheed M, Faizi S (2016) GC-MS profile of antimicrobial and antioxidant fractions from Cordia rothii roots. Pharma Biol 54(11):2597-2605. https ://doi.org/10.3109/13880209.2016.1172320

Kim TK, Chen J, Li W, Zjawiony J, Miller D, Janjetovic Z, Tuckey RC, Slominski A (2009) A new steroidal 5,7-diene derivative, 3-betahydroxyandrosta-5,7-diene-17beta-carboxylic acid, shows potent anti-proliferative activity. Europe PMC 75(3):230-239. https:// doi.org/10.1016/j.steroids.2009.12.004

Kim SS, Kim JE, Hyun CGu, Lee NH (2011) Neolitsea aciculate essential oil inhibits drug-resistant skin pathogen growth and Propionibacterium acnes-induced inflammatory effects on human monocyte leukemia. Nat Prod Com 6(8):1193-1198

Kondo S, Mamada A, Yamaguchi J, Fukuro S (1990) Protective effect of DL-alpha-tocopherol on the cytotoxicity of ultraviolet $\mathrm{B}$ against human skin fibroblasts in vitro. Europe PMC 7(4):173-177

Kumar D, Arora S, Verma A (2013) Fatty acid composition and antimicrobial and antioxidant activity of Cassla glauca seed extracts. Int J Phytopharm 4(2):113-118 
Kumar R, Deka CB, Roy RA (2012) Evaluation of orchid species under sub-tropical mid-hills of Meghalaya. HortFlora Res Spect 1(1):24-28

Kumar M, Kumar D, Raj V (2017) Studies on Imidazole and its derivatives with particular emphasis on their chemical/biological applications as bioactive molecules intermediated to bioactive molecules. Curr Synth Syst Biol 5(1):1-10. https://doi. org/10.4172/2332-0737.1000135

Kumar PP, Kumaravel S, Lalitha C (2010) Screening of antioxidant activity, total phenolic s and GC-MS study of Vitex negundo. Afri J Biochem Res 4(7):191-195

Kumar NN, Ramakrishnaiah H, Krishna V, Deepalakshmi PA (2015) GC-MS analysis and antimicrobial activity of seed oil of Broussonetia papyrifera (L.) Vent. Int J Pharm Sci Res 6:3954-3960

Kumaravel S, Bhabh PK, Singaravadivel K (2016) GC-MS and FT-IR analysis of the spice ajwain (Trachyspermum ammi). Int J Mod Res Rev 4(3):1114-1120

Kumaravel S, Muthukumaran P, Thomas N (2019) Phytochemical, GC-MS and FT-IR analysis of Papver somniferum L. J Pharm Biol Sci 7(1):1-8

Lakshmi VTP, Rajalakshmi P (2011) Identification of phyto-components and its biological activities of Aloe vera through the gas chromatography-mass spectrometry. Int Res J Pharm 2(5):247-249

Lalithi S, Parthipan B, Mohan VR (2015) Determination of bioactive components of Psychotria nilgiriensis Deb \& Gang (Rubiaceae) by GC-MS analysis. Int J Pharm Phytochem Res 7(4):802-809

Lawal B, Shittu OK, AbdulRasheed-Adeleke T, Ossai PC, Ibrahim AM (2015) Determination of bioactive constituents of giant African snail (Archachatina maginata) Haemolymph. J Pharm Biol Sci 10(2):59-64

Lindley, Paxton (1851) Pleione maculata. Flora of China 25: 326327. http://www.efloras.org/florataxon.aspx?flora_id=2\&taxon id $=242424455$.

Lipinski CA, Lombardo F, Dominy BW, Feeny PJ (1997) Experimental and computational approaches to estimate solubility and permeability in drug discovery and development setting. Adv Drug Del Rev 23(1-3):3-25. https://doi.org/10.1016/S0169 $-409 X(96) 00423-1$

Maiti S, Banerjee A (2020) Epigallocatechin gallate and theaflavin gallate interaction in SARS-COV-2 spike-protein central channel with reference to the hydroxychloroquine interaction: bioinformatics and molecular docking study. Drug Dev Res. https://doi. org/10.1002/ddr.21730

Mashiach E, Schneidman-Duhovny D, Andruisier N, Nussinov R, Wolfson HJ (2008) FireDock: a web server for fast interaction refinement in molecular docking. Nucl Acids Res 36:w229w232. https://doi.org/10.1093/nar/gkn186

Mohammad H, Prabhu K, Rao MRK, Sundram L, Dinakar S, Kumar MS, Vijayalakshmi N (2019) The gas chromatography-mass spectrometry study of one Ayurvedic pain relieving oil 'Karpooradi Thailam.' Drug Invent Today 12(7):1542-1546

Muthukrishnan S, Thinakaran R (2012) Chemical investigation of Pseudarthria viscida root by GC-MS analysis. Pharmacog Comm 2(3):26-29. https://doi.org/10.5530/pc.2012.3.6

Naidu RJ, Ismail BR, Yeng C, Sasidharan S, Kumar P (2012) Chemical composition and antioxidant activity of the crude methanolic extracts of Mentha spicata. J Physio 4(1):13-18

Oduje AA, Awode A, Edah A, Sagay I (2015) Characterization and phytochemical screening of Hexane oil extract from Cissus aralioides seeds. Int J Sci Eng Res 6(1):112-116

Okereke SC, Ijeh II, Arunsi UO (2017) Determination of bioactive constituents of Rauwolfia vomitoria Afzel (Asofeyeje) roots using gas chromatography-mass spectrometry (GC-MS) and Fourier transform infrared spectrometry (FT-IR). As J Pharm Pharmacol 11(2):25-31. https://doi.org/10.5897/AJPP2016.4712
Otaibi AA, McCluskey A (2013) Multicomponent reactions in Ionic liquids. Chapter-18, Ionic Liquids-New aspects for the future. IMTECH, pp 458-498

Padma M, Ganesan S, Jayaseelan T, Azhagumadhavan S, Sasikala P, Senthikumar S, Mani P (2018) Phytochemical screening and GC-MS analysis of bioactive compounds present in ethanolic leaves extract of Silybum marianum (L.). J Drug Del Therap 9(1):85-89

Palakkal L, Hukuman NHZ, Mullapally J (2017) Anti-oxidant activities and chemical composition of various crude extracts of Lepidagathis keralensis. J App Pharm Sci 7(6):182-189. https://doi. org/10.7324/JAPS.2017.7062

Panda PK, Arul MN, Patel P, Verma SK, Luo W, Rubahn HG, Mishra YK, Suar M, Ahuja R (2020) Structure-based drug designing and immunoinformatics approach for SARS-COV-2. Sci Adv 6:1-14

Pant B (2003) Medicinal orchids and their uses: tissue culture a potential alternative for conservation. Afri J Plant Sci 7(10):445-467. https://doi.org/10.5897/AJPS2013.1031

Pantsar T, Poso A (2018) Binding affinity via docking: fast and fiction. Molecules 23(1899):1-11. https://doi.org/10.3390/molec ules23081899

Paulpriya K, Tresina PS, Mohan VR (2014) Assessment of bioactive constituents by GC-MS of Crotalaria longipes Wight \& Arn: An endemic plant. Int J Pharm Phytochem Res 6(4):1043-1048

Pereira DM, Correia-da-silva G, Valentao P, Teixeira N, Andrade PB (2014) Anti-inflammatory effect of unsaturated fatty acid acids and Ergosta-7,22-dien-3-ol from Marthasterias glacialis. Prevention of CHOP-Mediated ER-stress and NF-kB activation. PLoS ONE 9(2):e88341. https://doi.org/10.1371/journal.pone.0088341

Pinto AEM, Araujo GS, Morais IM, Sa PN, Lima MC, Rosa AC, Siqueira PE, Johann S, Lima SRAL (2017) Antifungal and antioxidant activity of fatty acid methyl esters from vegetable oils. Ann Braz Acad Sci 89(3):1671-1681. https://doi.org/10.1590/0001-37652 0172016090

Plainfosse H, Burger P, Azoulay S, Landreau A, Verger-dubois G, Fernandez X (2017) Development of a natural-age ingredient based on Quercus pubescens wild leaves extract-a case study. Cosmetics 1(15):1-22. https://doi.org/10.3390/cosmetics5010015

Prabhadevi V, Sathish S, Johnson M, Venkatramani B, Janakiraman N (2012) Phytochemical studies on Allamanda cathartica L. using GC-MS. As Pac J Trop Med S550-S554

Prabhu DS, Rajeswari VD (2016) In silico docking analysis of bioactive compounds from chinese medicine Jinqi Jiangtang Tablet (JQJTT) using Patch Dock. J Chem Pharm Res 8(5):15-21

Qamar MTU, Alqahtani SM, Alamri MA, Chen LL (2020) Structural basis of SARS-CoV-2 3CLpro and anti-COVID-19 drug discovery from medicinal plants. J Pharm Anal. https://doi. org/10.1016/j.jpha.2020.03.009

Qurzeddine W, Fadel H, Mechehoud Y, Chalchat JC, Figueredo G, Chalard P, Benayache F, Benayache S (2017) Chemical composition and antioxidant activity of the fruit essential oil of Zizyphus lotus (L.) Desf. (Rhamanaceae). Int J Pharma Phytochem Res 9(2):228-232

Rajalakshmi K, Mohan VR (2016) Determination of Bioactive components of Myxopyrum serratulum A. W. HILL (Oleaceae) stem by GC-MS analysis. Int Res J Pharm 38(1):30-35

Rajashyamala G, Elango V (2015) Identification of bioactive components and its biological activities of Evolvulus alsinoides linn: a GC-MS study. Int J Chem Stud 3(1):41-44

Rajendra P, Bharathidasan R, Sureshkumar K (2017) GC-MS analysis of phyto-components in raw and treated sugarcane juice. Int $\mathrm{J}$ Curr Microbiol App Sci 6(7):51-61. https://doi.org/10.20546/ ijcmas.2016.501.007

Rajeswari G, Murugan M, Mohan RV (2013) GC-MS analysis of bioactive components of Hugonia mystax L. bark (Linaceae). J Pharm Biomed Sci 29(29):818-824 
Rane JS, Chatterjee A, Kumar A, Ray S (2020) Targeting SARSCoV-2 spike protein of COVID-19 with naturally occurring phytochemicals: an In silico study for drug development. ChemRxiv. https://doi.org/10.26434/chemrxiv.12094203.v1

Rautela I, Sharma MD, Sharma N, Kishor K, Singh K, Sharma N (2018) Comparative GC-MS analysis of leaf and root extract of medicinal plant Withania somnifera. World J Pharm Res 7(2):956-972

Ren R, Hashimoto T, Mizuno M, Takigawa H, Yoshida M, Azuma T, Kanazawa K (2013) A lipid peroxidation product 9-oxononanoic acid induces phospholipase A2 activity and thromboxane A2 production in human blood. J Clin Biochem Nutri 52(3):228-233. https://doi.org/10.3164/jcbn.12-110

Rizvi SI, Mishra N (2013) Traditional Indian medicines used for the management of Diabetes Mellitus. J Diab Res10.115/2013/712092

Sadler JJ, Smith MJ, Zettler WL, Alborn TH, Richardson WL (2011) Fragrance composition of Dendrophylax lindenii (Orchidaceae) using a novel technique applied in situ. Euro J Env Sci 1(2):137-141. https://doi.org/10.14712/23361964.2015.56

Saeidnia S, Manayi A, Gohari AR, Abdollahi M (2014) The story of Beta-sitosterol—a review. Euro J Med Plnt 4(5):590-609. https://doi.org/10.9734/EJMP/2014/7764

Sahin N, Kula I, Erdogan Y (2006) Investigation of antimicrobial activities of nonanoic acid derivatives. Fresenius Environ Bull 15(2):141-143

Sardar R, Satish D, Birla S, Gupta D (2020) Comaprative analyses of SARS-CoV2 genomes from different geographical locations and other coronavirus family genomes reveals unique features potentially consequential to host-virus interaction and pathogenesis. BioRxiv. https://doi.org/10.1101/2020.03.21.001586

Sathiyabalan B, Packia LM, Muthukumarasamy S, Mohan VR (2014) GC-MS Analysis of bioactive components of Petiveria alliacea L. whole plant (Phytolaccaceae). Int J Pharm Res Health Sci 2(5):387-392

Saxena M, Faridi U, Srivastava SK, Darokar MP, Mishra R, Pal A, Shisodia B, Khanuja SPS (2007) A cytotoxic and Hepatoprotective agent from Withania somifera and biological evaluation of its Ester derivatives. Nat Prod Comm 2(7):775-778. https:// doi.org/10.1177/2F1934578X0700200714

Schneidman-Duhovny D, Inbar Y, Nussinov R, Wolfson HJ (2005) PatchDock and SymmDock: servers for rigid and symmetric docking. Nucl Acids Res 33:W363-367. https://doi. org/10.1093/nar/gki481

Selvan PS, Velavan S (2015) Analysis of bioactive compounds in methanol extracts of Cissus vitiginea leaf using GC-MS technique. Rasayan J Chem 8(4):443-447

Sharad S (2019) Antisense therapy: an overview. Henry Jackson foundation for the advancement of military medicine, Bethesda

Sharma V, Chitranshi N, Agarwal AK (2014) Significance and biological importance of pyrimidine in the microbial world. Int $\mathbf{J}$ Med Chem 2014:1-31. https://doi.org/10.1155/2014/202784

Shen M, Zhou Y, Ye J, Al-maskri AAA, Kang Y, Zeng S, Cai S (2020) Recent advances and perspectives of nucleic acid detection for coronavirus. J Pharm Anal. https://doi.org/10.1016/j. jpha.2020.02.010

Shree LR (2012) Efficacy of supplementation of flaxseed and soybean on post-operative cancer patients. Thassim Beevi Abdul Kader College for Women, Chennai

Shweta M, Rashmi D (2019) In-vitro ADME studies of TUG-891, a GPR-120 inhibitor using Swiss ADME. J Drug Del Therap 9(2):266-369. https://doi.org/10.22270/jddt.v9i2-s.2710

Singh K, Kaur T (2016) Pyrimidine-based antimalarials: design stragtegies and antiplasmodial effects. MedChem Comm. https://doi. org/10.1039/C6MD00084C
Singh S, Srivastava HK, Kishor G, Singh H, Agrawal P, Raghava GPS (2017) Evaluation of protein-ligand docking methods on peptideligand complexes for docking small ligands to peptides. BioRxiv. https://doi.org/10.1101/212514

Singh AK (2016) Structure, synthesis and application of nanoparticles. Engineered nanoparticles, Structure, properties and mechanisms of toxicity

Subavathy P, Thilaga RD (2016) GC-MS analysis of bioactive compounds from whole body tissue methanolic extract of Cypraea arabica (L.1758). World J Pharma Res 5(3):800-806

Suffo AKL, Ashish R, Tedonkeng PE, Kuiate JR (2016) Effect of processing methods on chemical composition and antioxidant activities of two Amaranthus Sp. Harvested in West Region of Cameroons. J Nutri Food Sci 6(2):1-9

Sujatha K, Sivakamasundari MC (2014) GC-MS analysis of phytocomponents and total anti-oxidant activity of hexane extract of Sinapis alba. Int J Pharm Chem Biol Sci 4(1):112-117

Sujatha K, Vijayalakshmi V (2013) Foliar application of Caulerpa racemosa seaweed extract as bio-stimulant for enhancement of growth and yield of blackgram (Vigna mungo L.). Int J Adv Res Tech 2(10):216-230

Sulthanabegam M, Palani S, Senthikumar B (2019) Phytoconstituents screening by GC-MS and evaluation of In-vitro Gastroprotective and antioxidant activity of Vulva lactuca. Int J Pharm Chem Biol Sci 9(2):939-950

Surana P, Singh S, Manokaran S, Reddy AHM (2018) The interactions between variants of GALT in Galactosemia: In silico approach. Int J Fund App Sci 7(3):14-18

Suzuki K (1993) Anti-obesity effect of cholest-4-en-3-one, an intestinal catabolite of cholesterol, on mice. J Nutri Sci Vit (Tokyo) 39(5):537-543. https://doi.org/10.3177/jnsv.39.537

Suzuki K, Shimizu T, Nakata T (1998) The cholesterol metabolite cholest-4-en-3one and its-oxo derivatives suppress body weight gain, body fat accumulation and serum lipid concentration in mice. Bioorg Med Chem Lett 8(16):2133-2138. https://doi. org/10.1016/S0960-894X(98)00362-X

Sympli HD, Paul S, Borah VV, Choudhury M (2019) Phytochemical and anti-bacterial activity of an endangered orchid, Pleione maculata (Orchidaceae) of Meghalaya. Int J Res Anal Rev 6(2):221-232. https://doi.org/10.1729/Journal.21437

Teoh ES (2016) Orchids of Asia. Times Editions/Marshall Cavendish Singapoore 367

Thomas E, Aneesh TP, Thomas DG, Anandan R (2013) GC-MS analysis of phytochemical compounds present in the rhizomes of Nervilia aragoana Gaud. As J Pharm Clin Res 6(3):68-74

Tian S, Hu W, Niu Li, Lui H, Xu H, Xiao S (2020) Pulmonary pathology of early-phase 2019 Novel Coronavirus (COVID-19) Pneumonia in two patients with lung cancer. J Thorac Oncol 1-5

Tsering J, Tam N, Tag H, Gogoi JB, Apang O (2017) Medicinal orchids of Arunachal Pradesh: a review. Bull Arunachal For Res 32(1 \& 2):1-16

Tufino C, Bernal C, Ottone C, Romero O, Illanes A, Wilson L (2019) Synthesis with immobilized lipases and downstream processing of Ascorbyl palmitate. Molecules 24(3227):1-13. https://doi. org/10.3390/molecules 24183227

Tyagi T, Agarwal M (2016) Phytochemical screening and GC-MS analysis of bioactive constituents in the ethanolic extract of Pista stratiotes L. and Eichhornia crassipes (Mart.) solms. J Pharm Phytochem 6(1):195-206

Ukil S, Laskar S, Roy NR (2015) Physicochemical characterization and antibacterial activity of the leaf oil of Crotalaria pallida Aiton. J Taib Univ Sci. https://doi.org/10.1016/j.jtusci.2015.07.001

Umaiyambigai D, Saravanakumar K, Raj AG (2017) Phytochemical profile and antifungal activity of leaves methanol extract from the Psydrax dicoccos (Gaertn) Teys. \& Binn. Rubiaceace family. Int 
J Pharma Phytochem Ethnomed 7: 53-6. https://doi.org/10.18052 /www.scipress.com/IJPPE.7.53.

Upgade A, Bhaskar A (2013) Characterization and medicinal importance of phytoconstituents of C. papaya from down South Indian region using Gas Chromatography and Mass Spectroscopy. As J Pharm Clin Res 6(4):101-106

Valiei M, Shafaghat A, Salimi F (2011) Chemical composition and antimicrobial activity of the flower and root hexane extracts of Althaea officinalis in Northwest Iran. J Med Plnts Res 5(32):6972-6976. https://doi.org/10.5897/FJMPR11.963

Vardanyan RS, Hruby VJ (2006) Digitoxin.Synthesis of essential drug. Synth Essent Drugs Cardiotonic Drugs. https://doi.org/https:// doi.org/10.1016/B978-044452166-8/50017-0

Vijayakumari J, Raj TLS (2019) GC-MS analysis of secondary metabolites from acetone and chloroform extract of Dicranopteris linearis (burm.F.)Underw. Int Res J Biol Sci 8(9): 39-43.

Vijisaral ED, Arumugam S (2014) GC-MS analysis of bioactive constituents of Indigofera suffruticosa leaves. J Chem Pharm Res 6(8):294-300

Wang L, Wang Y, Ye D, Liu Q (2020) Review of the 2019 novel coronavirus (SARS-CoV-2) based on current evidence. Int J Antimicrob Agent 105948:1-7. https://doi.org/10.1016/j.ijantimicag.2020

Wei SL, Wee W, Siong FYJ, Syamsumir FD (2011) Characterization of antimicrobial, antioxidant, anticancer property and chemical composition of Michelia champaca seed and flower extract. Stam J Pharm Sci 4(1):19-24. https://doi.org/10.3329/sjps.v4i1.886

Wen C, Shyur L, Jan J, Liang P, Kuo C, Arulselvan P, Wu J, Yang N (2011) Traditional chineses medicine herbal extracts of Cibotium barometz, Gentiana scabra, Dioscorea batatas, Cassia tora, and Taxillus chinensis inhibit SARS-COV replication. J Trad Compl Med 1(1):41-50. https://doi.org/10.1016/s2225-4110(16)30055 $-4$

World Health Organization (WHO) (2018) Critical review report: Pregabalin. Expert committee on Drug Dependence forty-first meeting, Geneva

Wu D, Wu T, Liu Q, Yang Z (2020) The SARS-CoV-2 outbreak: what we know. Int J Inf Dis. https://doi.org/10.1016/j.ijid.2020.03.004

Wu Q, Zhou L, Sun X, Yan Z, Hu C, Wu J, Xu L, Li X, Liu H, Yin P, Zhao J, Li Y, Wang X, Li Y, Zhang Q, Xu GCH (2017) Altered lipid metabolism in recovered SARS patients twelve years after infection. Sci Rept 7:1-12. https://doi.org/10.1038/s41598-01709536-z

Yadav S, Pandey SK, Singh VK, Goel Y, Kumar A, Singh SM (2017) Molecular docking studies of 3-bromopyruvate and its derivaties to metabolic regaltory enzymes: Implication in deisgning of novel anti-cancer therapeutic strategies. PLoS ONE 2017:1-15. https://doi.org/10.1371/journal.pone.0176403.g005
Yang Y, Islam MS, Wang J, Li Y, Chen X (2020) Traditional Chinese medicine in the treatment of patients infected with 2019-New Coronavirus (SARS-CoV-2): a review and perspective. Int J Biol Sci 16(10):1708-1717

Yang P, Wang X (2020) COVID-19: a new challenge for human beings. Cell Mol Immunol 1-3

Yusufzai SK, Khan MS, Hanry EL, Rafatullah M, Elison BB (2019) GC-MS analysis of chemical constituents and in-vitro antioxidant activity of the organic extracts from the stem of Bridelia stipularis. Sans Malaysiana 48(5):999-1009. https://doi.org/10.17576 /JSM-2019-4805-08

Yuvaraj N, Arul V (2014) In vitro anti-tumor, anti-inflammatory, antioxidant, and antibacterial activities of marine brown alga Sargassum wightii collected from Gulf of Mannar. Glob J Pharm 8(4):566-577. https://doi.org/10.5829/idosi.gjp.2014.8.4.84281

Zafar MZ (2016) A case study: pnuemonia. Occu Med Health Aff 4(4):1-3

Zaini RG, Brandt K, Clench MR, Le Maire CL (2012) Effects of bioactive compounds from carrots (Daucus carota L.), Polyacetylenes, Beta-carotene and Lutein on human lymphoid leukaemia cells. Anti-canc Agnts Med Chem 12:1-13. https://doi. org/10.2174/187152012800617704

Zhang C, Vasmatzis G, Cornette JL, DeLisi C (1997) Determination of atomic desolvation energies from the structures of crystallized proteins. J Mol Bio 267(3):707-726. https://doi.org/10.1006/ jmbi.1996.0859

Zhang J, Sun H, Chen H, Chen S, Li Zeng, Wang T (2017) Anti-fungal activity, mechanism studies on $\alpha$ - Phellandrene and nonanal against Penicillium cyclopium. Bot Stud 58(13): 1-9. https://doi. org/10.1186/2Fs40529-017-0168-8

Zhang H, Penninger JM, Li Y, Zhong N, Slutsky AS (2020a) Angiotensin-converting enzyme 2 (ACE2) as a SARS-CoV-2 receptor: molecular mechanisms and potential therapeutic target. Inten Care Med 46:586-590

Zhang Z, Wu WY, Hou JJ, Zhang LL, Li FF, Gao L, Wu XD, Shi JY, Zhang R, Long HI, Lei M, Wu WY, Guo DA, Chen KX, Hofman LA, Ci ZH (2020b) Active constituents and mechanisms of respiratory detox shot, a traditional chinese medidine prescription, for COVID-19 control and prevention: network-molecular docking LC-MS analysis. J Int Med. https://doi.org/10.1016/j. joim.2020.03.00

Publisher's Note Springer Nature remains neutral with regard to jurisdictional claims in published maps and institutional affiliations. 